\title{
Morphological and Biophysical Determinants of the Intracellular and Extracellular Waveforms in Nigral Dopaminergic Neurons: A Computational Study
}

\author{
- $L$ Luciana López-Jury, ${ }^{1,2}$-Rodrigo C. Meza, ${ }^{1,3}$ Matthew T.C. Brown, ${ }^{4} \odot$ Pablo Henny, ${ }^{1 \star}$ and ${ }^{\circledR C}$ Carmen C. Canavier ${ }^{2 \star}$ \\ ${ }^{1}$ Laboratorio de Neuroanatomía, Departamento de Anatomía, and Centro Interdisciplinario de Neurociencia, NeuroUC, Escuela de Medicina, Pontificia \\ Universidad Católica de Chile, Santiago, 8330023, Chile, ${ }^{2}$ Department of Cell Biology and Anatomy and the Alcohol and Drug Abuse Center of Excellence, \\ Louisiana State University Health Sciences Center, New Orleans, Louisiana 70112, ${ }^{3}$ Centro Interdisciplinario de Neurociencia de Valparaíso CINV and \\ Millennium Nucleus of Biology of Neuropsychiatric Disorders NuMIND, Facultad de Ciencias, Universidad de Valparaíso, Valparaíso, Chile 2360102, and \\ ${ }^{4}$ Medical Research Council Anatomical Neuropharmacology Unit, University of Oxford, Oxford OX1 3TH, United Kingdom
}

Action potentials (APs) in nigral dopaminergic neurons often exhibit two separate components: the first reflecting spike initiation in the dendritically located axon initial segment (AIS) and the second the subsequent dendro-somatic spike. These components are separated by a notch in the ascending phase of the somatic extracellular waveform and in the temporal derivative of the somatic intracellular waveform. Still, considerable variability exists in the presence and magnitude of the notch across neurons. To systematically address the contribution of AIS, dendritic and somatic compartments to shaping the two-component APs, we modeled APs of previously in vivo electrophysiologically characterized and 3D-reconstructed male mouse and rat dopaminergic neurons. A parsimonious two-domain model, with high (AIS) and lower (dendro-somatic) $\mathrm{Na}^{+}$conductance, reproduced the notch in the temporal derivatives, but not in the extracellular APs, regardless of morphology. The notch was only revealed when somatic active currents were reduced, constraining the model to three domains. Thus, an initial AIS spike is followed by an actively generated spike by the axon-bearing dendrite (ABD), in turn followed mostly passively by the soma. The transition from being a source compartment for the AIS spike to a source compartment for the ABD spike satisfactorily explains the extracellular somatic notch. Larger AISs and thinner ABD (but not soma-to-AIS distance) accentuate the AIS component. We conclude that variability in AIS size and ABD caliber explains variability in AP extracellular waveform and separation of AIS and dendro-somatic components, given the presence of at least three functional domains with distinct excitability characteristics.

Key words: basal ganglia; dendrites; initial segment; pacemaking

\section{Significance Statement}

Midbrain dopamine neurons make an important contribution to circuits mediating motivation and movement. Understanding the basic rules that govern the electrical activity of single dopaminergic neurons is therefore essential to reveal how they ultimately contribute to movement and motivation as well as what goes wrong in associated disorders. Our computational study focuses on the generation and propagation of action potentials and shows that different morphologies and excitability characteristics of the cell body, dendrites and proximal axon can explain the diversity of action potentials shapes in this population. These compartments likely make differential contributions both to normal dopaminergic signaling and could potentially underlie pathological dopaminergic signaling implicated in addiction, schizophrenia, Parkinson's disease, and other disorders.

\section{Introduction}

Action potentials (APs) are the fundamental unit of neuronal signaling. AP shape is a key determinant of $\mathrm{Ca}^{2+}$ influx affecting presynaptic transmitter release (Jackson et al., 1991) and neural firing patterns (Liu et al., 2017). In dopaminergic (DA) neurons of the substantia nigra, which play a critical role in movement and motivation, the AP shape affects the dendritic release of dopa- 
mine (Beckstead et al., 2004; Ford et al., 2010) and dendritic $\mathrm{Ca}^{2+}$ influx mediating postsynaptic plasticity (Harnett et al., 2009). The relative timing of the AP in different regions of the neuron likely impacts both presynaptic and postsynaptic processes in complex ways.

Because of the technical difficulty of obtaining intracellular recordings, extracellular recordings are often performed when recording in vivo. Extracellular recordings are possible because completion of axial current circuits in nonisopotential neurons requires current flow in the extracellular medium (Rall and Shepherd, 1968; Johnston and $\mathrm{Wu}, 1995)$. Extracellular APs (EAPs) are recorded by moving the electrode tip closer to the soma of the recorded neuron than to that of any other neuron (Buzsáki, 2004) because the largest current densities are expected near the soma. EAP waveforms are used to identify neuronal classes (Csicsvari et al., 1999) and specific neurons in multiunit recordings (Rey et al., 2015). Several factors determine the shape of the EAP, including neural morphology, ion channel distribution, distance and orientation relative to the recording site, and properties of the extracellular medium (Gold et al., 2006). Mechanisms of EAP generation are also relevant to spike sorting algorithms requiring a detailed model of EAP generation (Telenczuk et al., 2018).

As in most central neurons (Bean, 2007), APs in DA neurons are initiated in the axon initial segment (AIS) (Grace and Bunney, 1983a, 1984; Grace, 1990). In these neurons, the AIS usually branches from a dendritic process $10-70 \mu \mathrm{m}$ from the soma in mice (Meza et al., 2018), and from a few micrometers to $240 \mu \mathrm{m}$ in rats (Juraska et al., 1977; Grace and Bunney, 1983a; Häusser et al., 1995; Blythe et al., 2009). Consequently, APs are observed in the axon-bearing dendrite (ABD) near the AIS before spreading to the soma and the rest of dendrites (Grace and Bunney, 1983a, 1984; Grace, 1990; Gentet and Williams, 2007). The somatic EAP often has two components. The first component (initial segment or AIS spike) is due to the influx of axial current from the distal locus of AP initiation. The second component (somatodendritic spike) likely has a more proximal locus. These components are separated by a notch or $\mathrm{V}$-shaped indentation slope (Grace and Bunney, 1983a, b). The shape of the first temporal derivative of the intracellular waveform, which also exhibits a notch, mimics the EAP (Grace and Bunney, 1983a, b; Ungless and Grace, 2012).

There is strong support in the literature for the twocomponent shape of the intracellular and extracellular AP in DA neurons (Grace and Bunney, 1983a, b; Kuhr et al., 1987; Bean, 2007; Henny et al., 2012; Tucker et al., 2012). However, there are studies in which the AIS component is either not observed or only seen in a fraction of neurons, as indicated by intracellular phase plane plots (Lerner et al., 2015), the first derivative of intracellular waveform (Gentet and Williams, 2007), or the extracellular AP (Brown et al., 2009; Meza et al., 2018). Indeed, we observed extracellular APs with two-component shape in rat dopaminergic neurons (Brown et al., 2009; Henny et al., 2012), but not in extracellular AP of mouse neurons (Meza et al., 2018) (see Fig. 1A). To gain insight into the mechanisms that explain the different

Correspondence should be addressed to either of the following: Dr. Carmen C. Canavier, Department of Cell Biology and Anatomy and the Alcohol and Drug Abuse Center of Excellence, Louisiana State University Health Sciences Center, New Orleans, LA 70112, E-mail: ccanav@Isuhsc.edu; or Dr. Pablo Henny, Laboratorio de Neuroanatomía, Departamento de Anatomía, and Centro Interdisciplinario de Neurociencia, NeuroUC, Escuela de Medicina, Pontificia Universidad Católica de Chile, Av. Libertador Bernardo 0'Higgins 340, Santiago, 8330023, Chile, E-mail: phenny@uc.cl.

M.T.C. Brown's present address: Wellcome Trust, Gibbs Building, 215 Euston Road, London NW1 2BE, United Kingdom.

DOI:10.1523/JNEUROSCI.0651-18.2018

Copyright $\odot 2018$ the authors $\quad 0270-6474 / 18 / 388296-16 \$ 15.00 / 0$
Table 1. Morphology of mouse and rat neurons

\begin{tabular}{lccc}
\hline & Mouse & Rat & \\
\hline Parameter & Value & Value & Units \\
AlS length & 41.6 & $37.0^{a}$ & $\mu \mathrm{m}$ \\
AlS surface & 88.9 & $47.9^{a}$ & $\mu \mathrm{m}^{2}$ \\
AlS distance to soma & 29.4 & $31.1^{a}$ & $\mu \mathrm{m}$ \\
Axon (AIS) origin branch order & 2 & 1 & \\
Mean ABD diameter & 2.71 & 1.17 & $\mu \mathrm{m}$ \\
Maximum ABD diameter & 4.25 & 1.55 & $\mu \mathrm{m}$ \\
Dendritic length & 6342.9 & 4189.6 & $\mu \mathrm{m}^{2}$ \\
Dendritic surface area & 12991.1 & 9108.3 & $\mu \mathrm{m}^{2}$ \\
Soma surface area & 1408.3 & 776.6 & $\mu \mathrm{m}^{2}$ \\
Soma volume & 4481.6 & 1131.1 & $\mu \mathrm{m}^{3}$ \\
No. of primary dendrites & 4 & 4 & \\
Highest branch order & 8 & 5 & \\
\hline
\end{tabular}

${ }^{a}$ Estimated values (see Materials and Methods).

components and observed diversity of EAP waveforms, we used multicompartmental models based on in vivo recorded EAP and real morphology of individual neurons to investigate the effects of morphological diversity in size and position of the AIS and ABD (Häusser et al., 1995; Blythe et al., 2009; González-Cabrera et al., 2017; Meza et al., 2018).

\section{Materials and Methods}

We modified an existing model of a DA neuron (Meza et al., 2018) implemented using the simulation program NEURON (Hines and Carnevale, 1997) (RRID:SCR_005393). Most simulations were run using a multicompartmental model neuron based on a real reconstructed morphology of a DA neuron from a male mouse SNc (Meza et al., 2018). The neuron, previously recorded and juxtacellularly labeled in vivo, was identified as dopaminergic by immunohistochemistry against TH and reconstructed using the Neurolucida (MBF Bioscience, RRID:SCR_001775) software (Henny et al., 2012, 2014; Meza et al., 2018). The morphology is shown in Figure $1 C$, and its dimensions are given in Table 1. The 3D coordinates and diameters thus obtained at the endpoints of each section were directly incorporated using the Import3D tool in the software package NEURON to convert morphometric data into the multicompartmental NEURON model, under the 3D specification of geometry that models each segment as a truncated cone. We also used an existing reconstruction of a male rat SNc DA neuron, recorded, labeled, and traced using similar methods and software (Henny et al., 2012, 2014) to test our model in a neuron with a different EAP shape (see Results) and morphology. The neuron dimensions are given in Table 1. All modeling parameters were identical for the mouse and rat models: they differed only in morphology.

Passive electrical properties, particularly membrane capacitance $\left(C_{M}\right)$ and axial resistance $\left(\mathrm{R}_{\mathrm{A}}\right)$, were modified from the original model (Meza et al., 2018) to better fit the typical experimental AP waveform and duration obtained from these neurons (Grace and Bunney, 1983a, b; Bean, 2007). The passive properties for the leak current $\left(\mathrm{I}_{\text {leak }}\right)$ were uniform for dendritic, somatic, and axonal membranes. The model also includes active conductances that generate spontaneous pacemaking activity. These active mechanisms include a voltage-dependent $\mathrm{Ca}^{2+}$ current $\left(\mathrm{I}_{\mathrm{Ca}}\right)$ (Cardozo and Bean, 1995; Durante et al., 2004) and a $\mathrm{Ca}^{2+}$ activated SK current $\left(\mathrm{I}_{\mathrm{SK}}\right)$ (Ping and Shepard, 1996). The fast $\mathrm{Na}^{+}$current $\left(\mathrm{l}_{\mathrm{Na}}\right)$ (Seutin and Engel, 2010; Ding et al., 2011b) initiates APs. Two types of $\mathrm{K}^{+}$ currents, including the noninactivating delayed rectifier $\left(\mathrm{I}_{\mathrm{Kdr}}\right)$ and the transient A-type $\left(\mathrm{I}_{\mathrm{KA}}\right)$, are involved in the repolarization phase (Silva et al., 1990; Gentet and Williams, 2007; Ding et al., 2011a). Parameters that were modified are summarized in Table 2. Otherwise, parameters are as in Meza et al. (2018). In addition, the kinetics of the $\mathrm{Na}^{+}$and $\mathrm{K}^{+}$current as well as the $\mathrm{Na}^{+}$and $\mathrm{K}^{+}$channel densities were modified from the previous model (Meza et al., 2018) as shown in Table 3 to better match the overall shape of both the intracellularly and extracellularly recorded AP waveforms reported by others (Grace and Bunney, 1983a, b; Bean, 2007; Ungless and Grace, 2012) and our own recordings (see Fig. 1A). In 
Table 2. Parameters that were modified from the previous model (Meza et al., 2018) ${ }^{a}$

\begin{tabular}{|c|c|c|c|c|}
\hline Parameter & Symbol & Region & Value & Units \\
\hline Axial resistivity & $\mathrm{R}_{\mathrm{a}}$ & Soma/dendrites/axonal & 100 & $\Omega-\mathrm{cm}$ \\
\hline Membrane capacitance & $C_{m}^{a}$ & Soma/dendrites/axonal & 1.0 & $\mu \mathrm{F} / \mathrm{cm}^{2}$ \\
\hline $\mathrm{Na}^{+}$conductance density & $g_{\mathrm{Na}}$ & Dendrites/axon & 800 & $\mu S / \mathrm{cm}^{2}$ \\
\hline $\mathrm{K}_{\mathrm{dr}}{ }^{+}$conductance density & $g_{K d r}$ & Dendrites/axon & 840 & $\mu S / \mathrm{cm}^{2}$ \\
\hline $\mathrm{Na}^{+}$conductance density & $g_{\mathrm{Na}}$ & Axon-initial segment & 4000 & $\mu \mathrm{S} / \mathrm{cm}^{2}$ \\
\hline $\mathrm{K}_{\mathrm{dr}}{ }^{+}$conductance density & $g_{K d r}$ & Axon-initial segment & 1680 & $\mu S / \mathrm{cm}^{2}$ \\
\hline L-type $\mathrm{Ca}^{2+}$ conductance density & $g_{\text {CaL }}$ & Soma/dendrites/axonal & 3.36 & $\mu S / \mathrm{cm}^{2}$ \\
\hline $\mathrm{SKK}^{+}$conductance density & $g_{S K}$ & Soma/dendrites/axonal & 11.8 & $\mu S / \mathrm{cm}^{2}$ \\
\hline A-type $\mathrm{K}^{+}$conductance density & $g_{K A}$ & Dendrites/axonal & 266 & $\mu S / \mathrm{cm}^{2}$ \\
\hline A-type $\mathrm{K}^{+}$conductance density & $g_{K A}$ & Soma & 570 & $\mu \mathrm{S} / \mathrm{cm}^{2}$ \\
\hline \multirow[t]{2}{*}{$\mathrm{Na}^{+}$conductance density } & $g_{\mathrm{Na}}$ & Soma & $800^{b}$ & $\mu S / \mathrm{cm}^{2}$ \\
\hline & & & 200 & \\
\hline \multirow[t]{2}{*}{$\mathrm{K}_{\mathrm{dr}}+$ conductance density } & $g_{K d r}$ & Soma & $840^{b}$ & $\mu S / \mathrm{cm}^{2}$ \\
\hline & & & $100^{c}$ & \\
\hline
\end{tabular}

${ }^{a}$ Axonal indicates axon initial segment and the rest of the axon reconstructed; axon does not consider the axon initial segment.

${ }^{b}$ Two-domain model.

Three domain model.

Table 3. Parameters for the equations describing the $\mathrm{Na}^{+}$current $^{a}$

\begin{tabular}{|c|c|c|c|c|}
\hline Variable & Parameter & Region & Value & Units \\
\hline \multirow[t]{3}{*}{$\mathrm{Na}^{+}$activation, $\mathrm{m}_{\infty}$} & $V_{\text {half }}$ & Soma/dendrites/axon & $v-30.0$ & $\mathrm{mV}$ \\
\hline & $S$ & Soma/dendrites/axonal & 9.0 & - \\
\hline & $V_{\text {half }}$ & Axon-initial segment & -33.0 & $\mathrm{mV}$ \\
\hline \multirow{3}{*}{$\mathrm{Na}^{+}$inactivation, $\mathrm{h}_{\infty}$} & $V_{\text {half }}$ & Soma/dendrites/axon & -35.0 & $\mathrm{mV}$ \\
\hline & $S$ & Soma/dendrites/axonal & -10.0 & - \\
\hline & $V_{\text {half }}$ & Axon-initial segment & -38.0 & $\mathrm{mV}$ \\
\hline \multirow[t]{2}{*}{$\mathrm{K}^{+}$activation, $\mathrm{n}_{\infty}$} & $V_{\text {half }}$ & Soma/dendrites/axonal & -25.0 & $\mathrm{mV}$ \\
\hline & $S$ & Soma/dendrites/axonal & 2.0 & - \\
\hline \multirow{2}{*}{$\begin{array}{l}\text { Activation, } \tau_{\mathrm{m}} \\
\text { Inactivation, } \tau_{\mathrm{h}}\end{array}$} & Additive constant & Soma/dendrites/axonal & 0.01 & $\mathrm{~ms}$ \\
\hline & Additive constant & Soma/dendrites/axonal & 0.4 & $\mathrm{~ms}$ \\
\hline
\end{tabular}

${ }^{a}$ Axonal indicates axon-initial segment and the rest of the axon reconstructed; axon does not consider the axon initial segment.

addition, the $\mathrm{Na}^{+}$current kinetics used in previous models (Kuznetsova et al., 2010; Meza et al., 2018) produced an additional, nonphysiological notch in the negative phase of the EAP (data not shown). The equations for the time constants of activation and inactivation of the fast sodium channel contain an additive constant that provides a floor, or minimum value for the time constant. To remove the negative notch, the floor for the activation was reduced from 0.04 to $0.01 \mathrm{~ms}$ and the floor for the inactivation was reduced from 1 to $0.4 \mathrm{~ms}$.

The active properties underlying the pacemaking and AP-generating currents were uniformly distributed throughout the somatodendritic tree, except the A-type current, which was heterogeneously distributed (Kuznetsova et al., 2010). Axonal compartments were assumed to have the same properties as dendrites, except the AIS. $\mathrm{Na}^{+}$channel density in this structure was fivefold higher than the rest of the neuron within the 3to 40-fold increases reported by others (Fleidervish et al., 2010; Bender and Trussell, 2012), and the voltage dependence of the activating and inactivating kinetics was uniformly shifted $3 \mathrm{mV}$ in the hyperpolarized direction. The delayed rectifier channel density in the AIS was increased by twofold. In the three-domain model (see Results), the maximum $\mathrm{Na}^{+}$ and $\mathrm{K}^{+}$channel conductances were reduced by a factor of 4 and 8.4 times, respectively, in the somatic compartment only.

The size and position of AIS (Fig. 1C, red) were previously determined experimentally using Ankyrin-G labeling on juxtacellularly labeled and neurochemically identified nigral DA neurons (Meza et al., 2018). Artificial modifications of size and length in the current study were performed using the experimentally observed range of variability in size and position of the AIS. Electrode recording location in relation to the soma or dendrites was documented for 14 neurons by examining the glass electrode track on the coronal sections that contained the neurobiotinstained neurons. The model reproduces the pacemaking activity typical of nigral DA neurons (see Fig. 1C, inset). Consistent with experimental observations in these neurons (Gentet and Williams, 2007), the AIS com- partment fires before the ABD and soma (see Fig. 1D). Staining for Ankyrin-G or other AIS markers was not performed on the rat neuron, so the length of the AIS could not be specified. We used instead the rule for the relationship of AIS length to its distance from the soma described previously (Meza et al., 2018) to estimate the length of the AIS in the rat neuron only. In contrast to the previous study (Meza et al., 2018), we incorporated the NEURON extracellular mechanism in our neuron model, retaining the default parameters for this mechanism. Most simulations of the extracellular potential in our study used the xtra mechanism downloaded from https://neuron.yale.edu/ftp/ted/neuron/extracellular_stim_and_rec.zip in the NEURON simulation package. This mechanism calculates the local field potential observed at a single point as a consequence of the membrane currents generated by all compartments of a single cell under the assumptions of linearity, negligible dispersion, negligible extracellular interactions between the model segments, and that the distance from the middle of each segment to the observation point is at least 10 times the segment length. To assess how heavily our results depended on the method used for simulating extracellular potentials, we also used LFPy (Lindén et al., 2014), an open source Python package for numerical simulations of extracellular potentials. LFPy runs on top of the NEURON simulation environment and also calculates the extracellular potential as a weighted sum of all transmembrane currents in all compartments located in the vicinity of the electrode, but uses an efficient implementation of the line source approximation method. Using LFPy, we simulated the basic shape of the extracellularly recorded AP, as well as the results obtained previously with xtra mechanism.

All analyses were performed on spontaneous APs generated during simulated pacemaking; we did not study spikes evoked from a hyperpolarized potential with a depolarizing current pulse or antidromically evoked spikes. To test how morphology affects the shape of intracellular and extracellular APs, we used the section length and diameter parame- 
A

Without notch
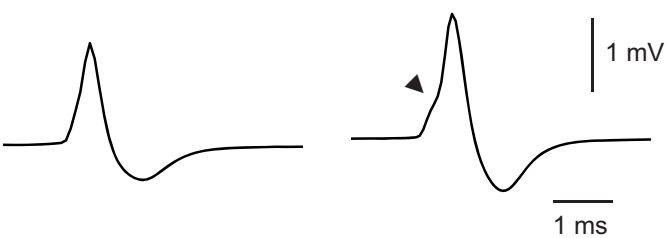

C

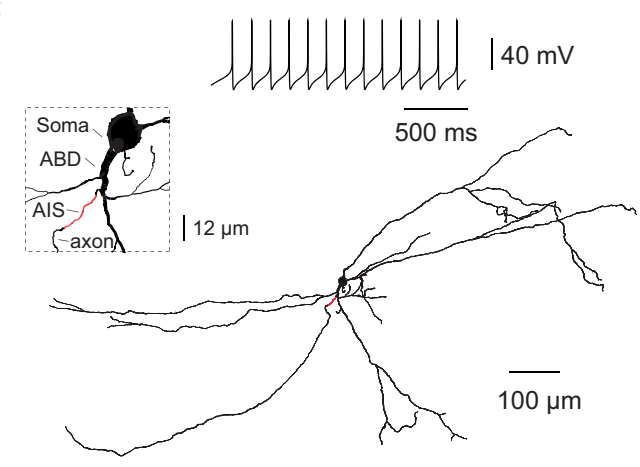

B

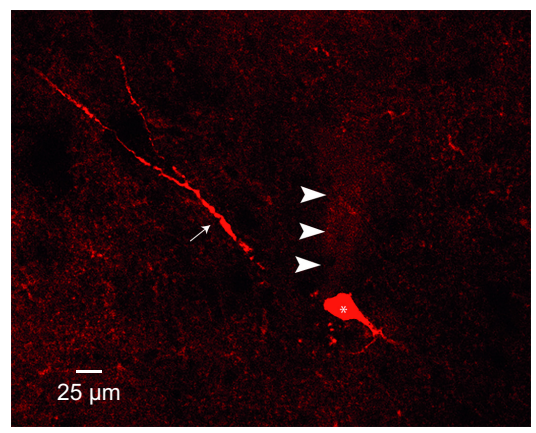

D

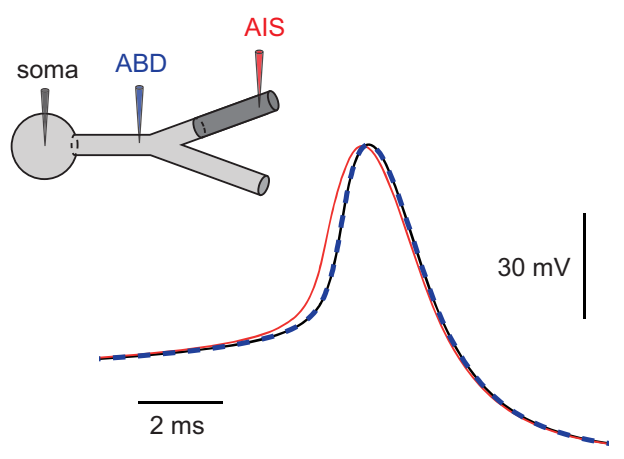

Figure 1. Different extracellular APs waveforms of nigral DA neurons and a computational model. $\boldsymbol{A}$, Experimental extracellularly recorded APs from SNc neurons without (left, from mouse) and with (right, from rat) a notch (arrowhead). B. Most of the electrodes (track indicated by three arrowheads) used to record extracellular APs and label cells (red, neurobiotin revealed with streptavidin-Cy3) recorded somatic activity $\left({ }^{*}\right)$. Arrow indicates a main dendritic trunk from the same individual neuron. $C$, Reconstructed morphology from nigral dopaminergic mouse neuron of extracellular AP shown in $\boldsymbol{A}$. The 3D morphological reconstruction, as traced using the Neurolucida software from an in vivo juxtacellularly labeled neuron (Meza et al., 2018), was implemented in the NEURON software. Black represents somatic, dendritic, and axonal compartments. Red represents AIS (experimentally identified for this neuron) (Meza et al., 2018). Left, Inset, Magnified view of the precise architectural arrangement. Top, Inset, Simulated pacemaking in the model. D, Schematic representation of the full mouse neuron model and the location of simulated intracellular electrodes. Bottom, Traces showing that simulated AP starts at the AIS, followed by the soma and ABD.

ters (L and diam) in NEURON to artificially modify the AIS and the $\mathrm{ABD}$. Unless otherwise specified, in this study, we considered the ABD as the dendritic portion between the soma and the AIS (Meza et al., 2018). The location of extracellular electrode was set using the $\mathrm{x} \_x t r a, y \_x t r a$, and z_xtra NEURON parameters that determine the 3D location of a particular compartment. We used the VariableTimeStep tool of NEURON to set the relative error tolerance at 0.001 for the adaptive integration method. AIS distance to soma was modified by lengthening the appropriate segment of the ABD.

\section{Results}

As mentioned earlier, extracellular APs waveforms vary among DA neurons. In a previous study (Meza et al., 2018), we found that the EAP of identified mouse SNc dopaminergic neurons did not exhibit a visible separation (notch) between two components (Fig. 1A). In contrast, our previous studies in rats (Brown et al., 2009; Henny et al., 2012) found that $\sim 40 \%$ of EAP from identified SNc dopaminergic neurons did exhibit a notch (Fig. 1A). We checked whether the location of the recording electrode in our sample of mice neurons could account for the absence of a notch. We were able to analyze the location of the electrode track in 14 neurons and found that 11 of them had been recorded near the cell body (Fig. $1 B$ ) and three from proximal dendritic locations, suggesting that the absence of a notch did not depend on electrode location.

\section{AIS contribution to intracellular APs}

We then hypothesized that the morphology of the DA neurons might determine whether a notch is observed in the extracellular recordings. This could potentially explain why we did not observe a notch on the experimental EAP (Fig. 1A). To systematically address this possibility, we started analyzing the effect of morphology on the intracellular waveform.

To predict the effects of morphology on somatic intracellular AP shape (Fig. 2), we compared somatic intracellular membrane potential (top row traces), its first temporal derivative (middle row), and phase plane plots (bottom row) from simulations in which we varied the position and size of the AIS, as well as the size of the ABD for the reconstruction shown in Figure $1 C$.

We found that changing the distance between soma and AIS (a modification that is equivalent to increasing ABD length, which in this study is the dendritic portion between soma and AIS; see Materials and Methods) with AIS length held constant, causes little perceptible change in the somatic intracellular traces of membrane potential, the temporal derivative, or phase plane plot (Fig. 2A). On the other hand, as the position of the AIS is held fixed relative to the soma and the length of the AIS is increased, a kink appears and becomes more prominent in the intracellular traces (Fig. 2B, arrow). The kink (Naundorf et al., 2006; Brette, 2013 ) is a steeper, more abrupt initial rising phase than would be observed without an axonal spike (McCormick et al., 2007; Yu et al., 2008), and it has been shown to reflect to distal initiation of the AP (Yu et al., 2008) in a compartment with a lower threshold (AIS). Changes in ABD diameter for values within the experimentally observed range had a modest effect on intracellular AP waveform (Fig. 2C).

In the corresponding first temporal derivatives (Fig. $2 C$, middle row) and phase plane plots (Fig. 2C, bottom row), a notch 
A

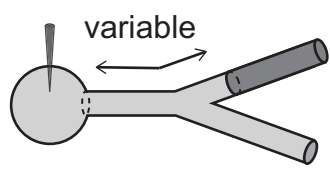

distance to soma $(\mu \mathrm{m})$

15
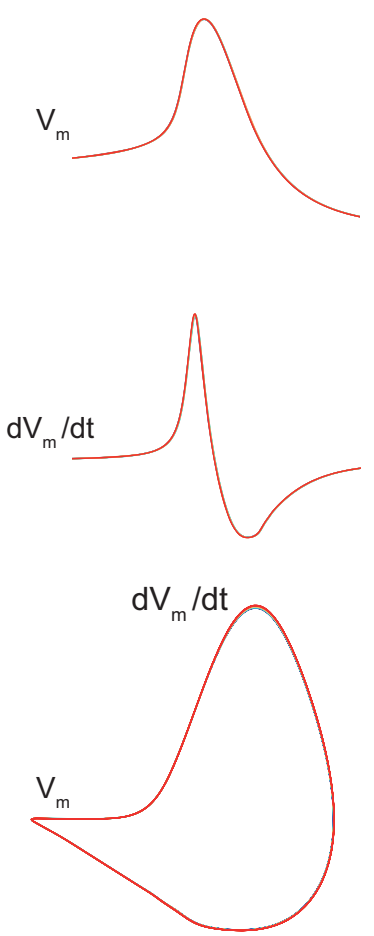

B
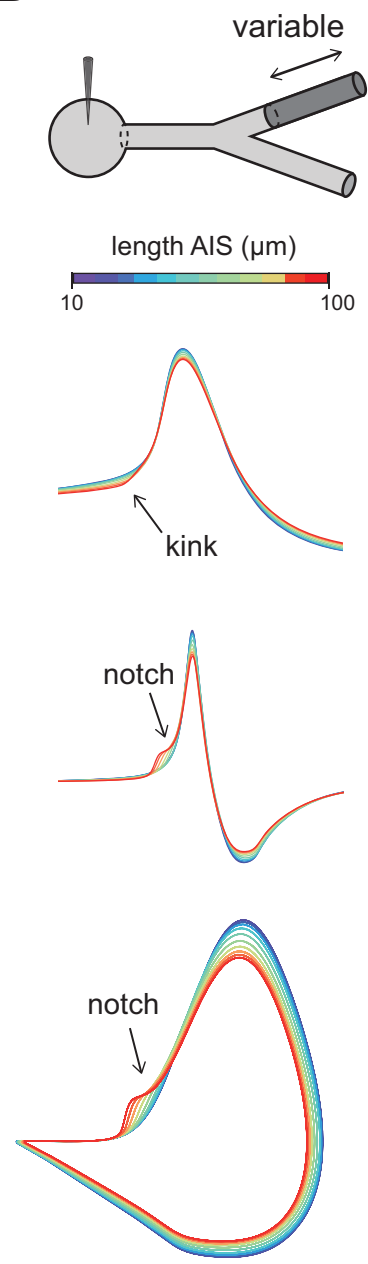

C
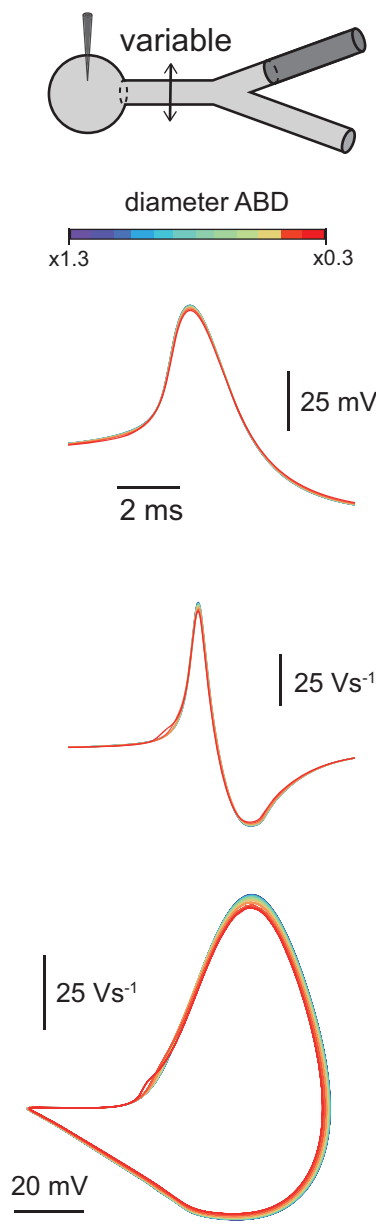

Figure 2. Predicted influence of AIS distance, AIS size, and ABD diameter on somatic intracellular AP shape. $A$, Varying the distance from soma to proximal border of AIS (equivalent to varying in ABD length, top and color code bar) from 15 to $60 \mu \mathrm{m}$ in increments of $5 \mu \mathrm{m}$ does not affect intracellular AP shape (top), first temporal derivative (middle), or the phase plane plot (bottom) recorded in the soma (top). AIS length held constant at its true value of $41.57 \mu \mathrm{m}$. ABD diameter tapered from 4.25 to 3.05 to $0.83 \mu \mathrm{m}$, as in original reconstruction. $\boldsymbol{B}$, Increasing AIS length (top and color code bar) from 10 to $100 \mu \mathrm{m}$ in increments of $10 \mu \mathrm{m}$ makes the kink more prominent in the intracellular potential (top, arrow). It also makes the AIS component and the notch that follows it more prominent in the first temporal derivative and phase plane plots (middle, bottom, arrows). Als distance held constant at $29.36 \mu \mathrm{m}$. ABD diameter tapered from 4.25 to 3.05 to $0.83 \mu \mathrm{m}$ as in original reconstruction. C, Decreasing ABD diameter (top and color code bar) from a factor of 1.3 to 0.3 in decrements of 0.1 makes the kink more prominent in the intracellular $A P$ (top). Decreasing $A B D$ diameter also makes the AIS component and notch more visible. AIS distance held constant at $29.36 \mu \mathrm{m}$ and AIS length held constant at $41.57 \mu \mathrm{m}$. Here, as in Figures $1-9$, all simulations are run in the real reconstructed neural morphology shown in Figure 1C, and tops are simply schematic diagrams for illustrative purposes.

(Fig. 2C, arrows) appears and becomes more prominent as AIS length is increased. The notch manifests as a decrease followed by an increase in slope and separates the AIS and dendro-somatic (DS) components of the AP. We favored the use of DS spike over somatodendritic spike as the ABD spike necessarily precedes the somatic spike in most neurons (Gentet and Williams, 2007). The notch is reduced and eventually disappears as the AIS becomes smaller, which is consistent with the difference observed between phase plots of recordings of these neurons in a slice preparation versus in cell culture, where the AIS is likely not present (Bean, 2007). Increasing the total AIS surface area by increasing diameter rather than the length of the AIS produces similar effects (data not shown). Finally, changes in ABD diameter had a modest but visible effect; decreasing the diameter of the $\mathrm{ABD}$ accentuated the notch in the temporal derivative and the phase plane plots (Fig. 2C).

In summary, increasing the length of the AIS in the reconstructed mouse model from its actual $(42 \mu \mathrm{m})$ to larger values resulted in a more prominent kink in intracellular traces and a more prominent notch in the first temporal derivative and phase plane plot. Increasing AIS distance and ABD diameter had no and modest effects, respectively.

\section{Determinants of the appearance of a notch in rising phase of the extracellular AP}

Having predicted the influence of AIS and ABD morphology in intracellular AP waveforms recorded in the soma, we tested whether AIS and ABD morphology could also influence the shape of the EAP waveform. The unfiltered EAP during spontaneous firing of DA neurons is generally biphasic (positive-negative) and, as mentioned before, can show a notch in the rising phase and a prominent negative component (Ungless and Grace, 2012). However, using the "Extracellular stimulation and recording" NEURON module (Carnevale and Hines, 2006), simulations of the extracellular recordings near the soma were triphasic and did not exhibit a notch, despite the presence of the notch in the first 
A

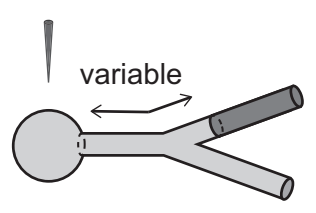

distance to soma $(\mu \mathrm{m})$

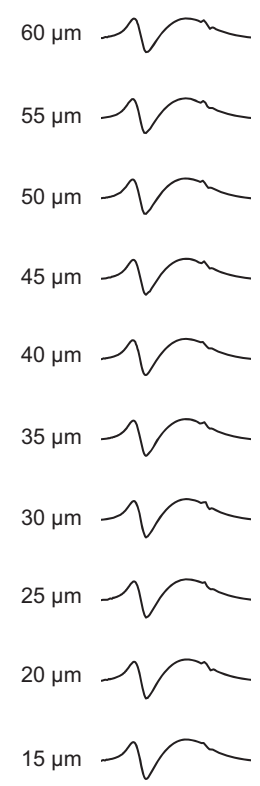

B

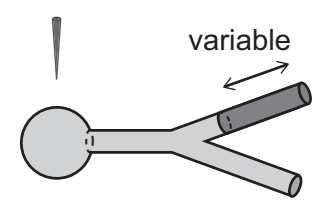

length AIS $(\mu \mathrm{m})$

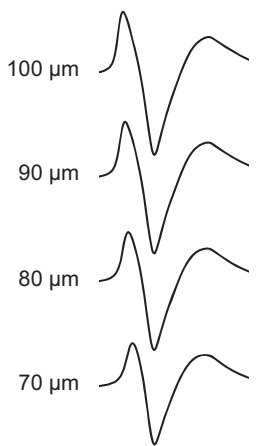

$60 \mu \mathrm{m}$
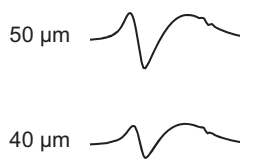

$30 \mu \mathrm{m}$
C

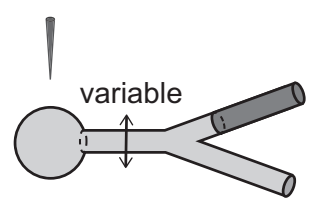

diameter $\mathrm{ABD}$

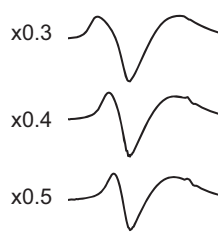

$x 0.6 \bigcirc$

$x 0.7 \bigcirc$

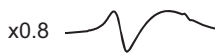

$x 0.9 \bigcirc$

$\times 1.0 \bigcirc$

$\times 1.1 \bigcirc$

$\times 1 . 2 \longdiv { }$

$\times 1.3 \checkmark$
D

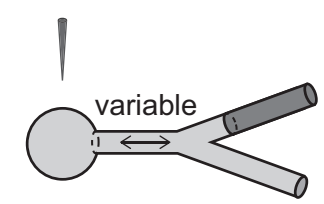

citoplasmic resistivity $(\Omega-\mathrm{cm})$

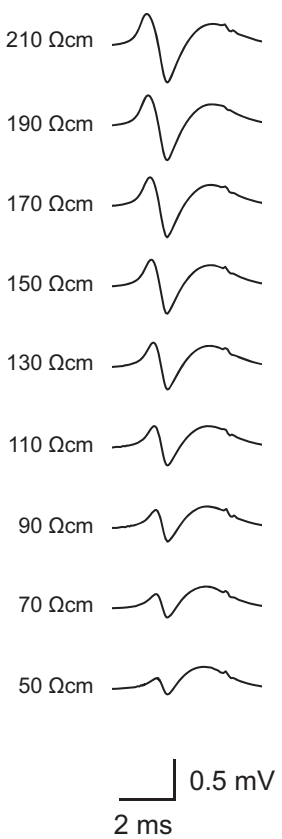

Figure 3. A two-component AP shape and notch in the somatic extracellular potential cannot be obtained by modifying AIS distance, AIS size, ABD diameter, or cytoplasmic resistivity. $\boldsymbol{A}$, Varying the distance from soma to proximal AIS (equivalent to varying ABD length, top) does not affect somatic extracellular AP shape. $\boldsymbol{B}$, Increasing AIS length (top) increases the amplitude of the extracellular AP, without producing a two-component waveform and its notch in the ascending phase. C, Decreasing ABD diameter (top), while producing evident changes in AP shape (broadening and increasing its amplitude), also does not generate a notch. $\boldsymbol{D}$, Increasing cytoplasmic resistivity also broadens the waveform and increases its amplitude. The resistivity tested was from 50 to 210 $\Omega$-cm in increments of $20 \Omega-\mathrm{cm}$.

temporal of the intracellular AP and in the temporal derivatives and phase plane plots (Fig. 2). Triphasic EAPs in these neurons are generally associated with overfiltering, and become biphasic when unfiltered. Thus, the triphasic EAPs observed in the model (where filtering is not present or modeled) are not consistent with the literature (Ungless and Grace, 2012).

We systematically varied morphological parameters within experimentally observed ranges (Meza et al., 2018) to determine whether any modification of the morphological parameter set could produce a separation of components in the EAP. Increasing the distance between soma and AIS with AIS length held constant (as in Fig. 2A, also equivalent to increasing ABD length) did not produce a notch in the EAP (Fig. $3 A$ ) nor a change in EAP amplitude. Increasing AIS length merely (though dramatically) increased the amplitude of the EAP, but it also did not produce a notch (Fig. $3 B$ ). Changes in AIS diameter reproduced the changes in AIS length (data not shown). Decreasing ABD diameter resulted in changes in EAP amplitude and duration but also did not produce a notch (Fig. 3C).

Because morphological variability was not sufficient to produce a notch in the EAP, we modified other biophysical parameters. Increasing $\mathrm{Na}_{\mathrm{v}}$ density in the AIS (data not shown) had the same effect as increasing AIS length. Increasing cytoplasmic axial resistivity increased EAP amplitude and duration (Fig. 3D) but, like all the other manipulations, did not produce a notch. In sum, although AIS and ABD morphology is clearly responsible for changing the amplitude and/or duration of the EAP, as well as the presence of the kink in somatic intracellular recordings and for the notch in the first derivative of the intracellular somatic AP and in phase plane plots (Fig. 2), we found no evidence that changes in AIS or ABD morphology alone could produce a notch in the somatic EAP (Fig. 3).

\section{Compartmentalization of spike generating domains of DA neurons: mathematical derivation}

As mentioned above, studies have shown that the EAP in DA neurons resembles the first derivative of intracellularly recorded somatic AP (Grace and Bunney, 1983a; Ungless and Grace, 2012). However, our simulated EAP did not resemble the first temporal derivative of the somatic membrane potential (compare dVm/dt from Fig. 2 with EAP from Fig. 3). We also found that, in contrast to experimental observations, our simulated EAPs were triphasic. The basis for the similarity between the extracellularly recorded AP waveform and the first temporal derivative of the membrane was previously derived and observed in spinal motoneurons (Brooks and Eccles, 1947; Fatt, 1957; Freygang and Frank, 1959; Terzuolo and Araki, 1961). We recapitulated here a semiquantitative deri- 

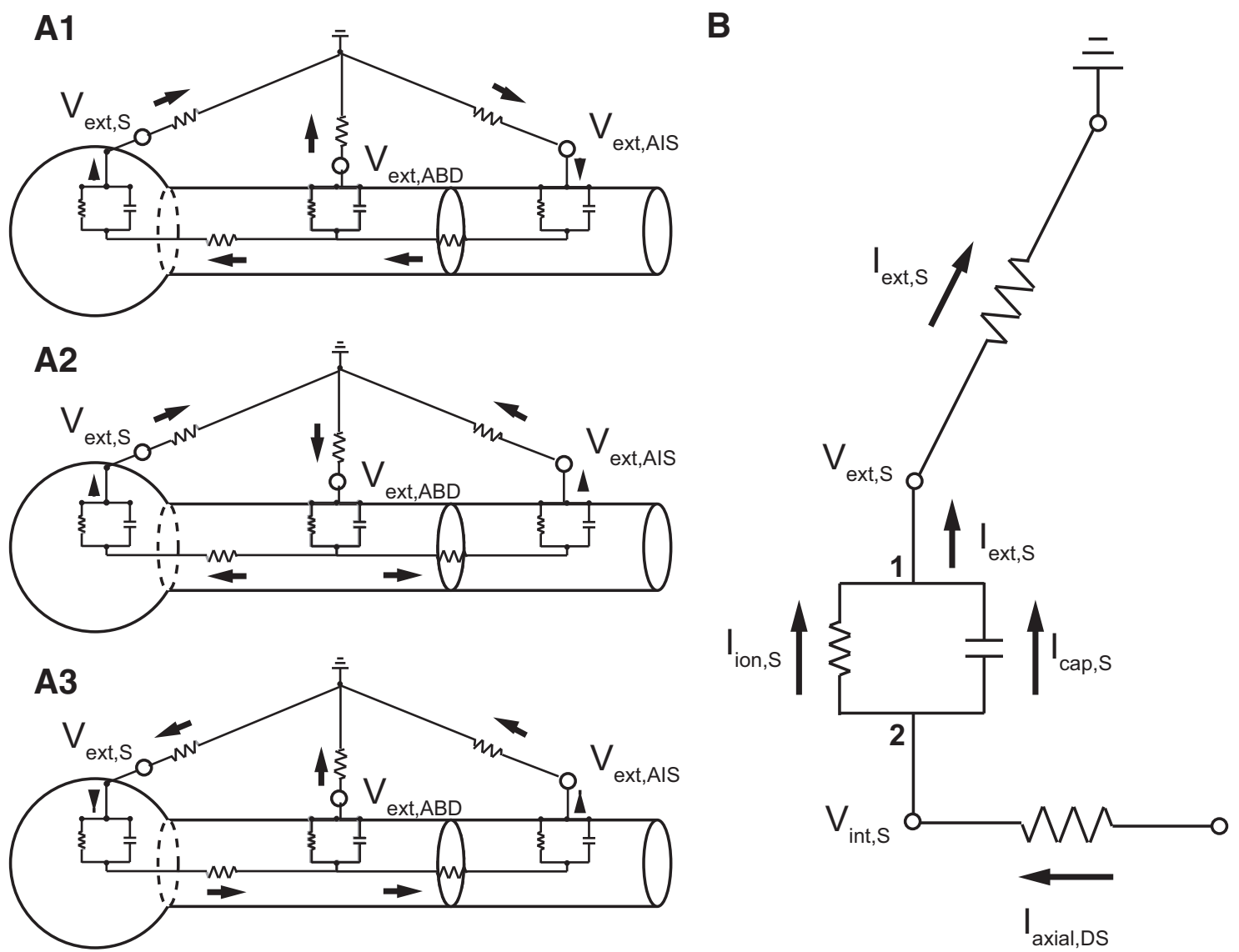

Figure 4. Hypothetical model of spike generation in DA neurons. $A$, Circuits are shown linking axial current flow between the three domains (soma, ABD, and AIS) to current sources and sinks relative to distant ground during the AIS component of the somatic EAP (A1), during the DS component of the somatic EAP (A2), and during the negative deflection of the somatic EAP (A3). Sinks and sources are determined by the sign of the extracellular arrow emanating from each domain, with an inward directing indication a sink and an outward direction indicating a source. Axially directed extracellular currents are neglected in this diagram. Open circles next to $\mathrm{V}_{\text {ext, }, \mathrm{S}} \mathrm{V}_{\text {ext,ABD}}$, and $\mathrm{V}_{\text {ext,AIs }}$ represent the points at which an electrode could be inserted to measure the extracellular potential near the soma, ABD, and AIS, respectively. The extracellular potentials are measured with respect to a distant ground (top). $\boldsymbol{B}$, Idealized circuit diagram of the somatic compartment used to derive the correspondence between the extracellularly recorded potential with reference to a distant ground and the first temporal derivative of the intracellularly recorded membrane potential.

vation (Johnston and $\mathrm{Wu}, 1995$ ) that the perisomatic extracellular potential is approximated by the first temporal derivative of the somatic intracellular potential provided the recording site is near the soma (Grace and Bunney, 1983a) and that, critically, the axial current flowing between the soma and the adjacent compartments overwhelms the contribution of ionic somatic membrane currents.

Figure 4 shows idealized circuit diagrams of the current flow during an AP between three compartments both intracellularly (axially) and through a distant ground in the extracellular space. Figure $4 A$ illustrates the three presumed functional compartments: the soma, the $\mathrm{ABD}$, and the AIS, with the extracellular potential being measured between a point near a compartment, assumed to be the soma (Grace and Bunney, 1983a), and a distant ground. Figure $4 B$ shows currents associated with the somatic compartment. A Kirchoff's current balance at point 2 in Figure $4 B$ gives $I_{\text {axial }, D S}=I_{c a p, s}+I_{i o n, s}$, and a current balance at point 1 gives $I_{c a p, s}+I_{i o n, s}=I_{e x t, S}$, where $I_{\text {ion }, S}$ is the ionic component of the somatic membrane current, $I_{c a p}, S$ is capacitive current that charges the somatic membrane, $I_{a x i a l, D S}$ is the current flowing axially from the $\mathrm{ABD}$ to the soma, and $I_{\text {ext }, S}$ is the current emanating from the somatic compartment into the extracellular space. It is clear from these equations that $I_{\text {ext, } S}=I_{a x i a l, D S}$. From the definition of the capacitive current, we obtain $I_{c a p, s}=C_{M} \frac{d V_{\text {int, }}}{d t}$, and sub- stitution into the current balance at point 2 and rearranging gives $I_{e x t, S}=I_{i o n, S}+C_{M} \frac{d V_{\text {int }, S}}{d t}$. To obtain a semiquantitative (Johnston and $\mathrm{Wu}, 1995$ ) relationship between the intracellular and extracellular potential, we must make the assumption that $I_{\text {axial, } D S} \gg I_{\text {ion, } S}$, which implies that $I_{\text {ext }, S} \gg I_{i o n, S}$ and that $I_{\text {ext }, S} \approx$ $C_{M} \frac{d V_{\text {int, } S}}{d t}$. The extracellular potential at any point in the extracellular space relative to a distal ground has contributions from the point sources on all compartments depending on the distance and geometry. However, if we assume that $I_{e x t, S}$ is the major contributor to $V_{\text {ext, } S}$, then by Ohm's law, $V_{\text {ext, } S} \approx I_{\text {ext, } S} R_{\text {ext, } S}$ and the somatic extracellular potential is proportional to the somatic

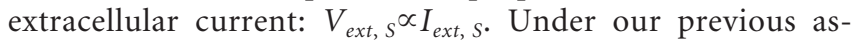
sumption that, during an AP, the currents flowing axially into the soma from the dendrites are far greater than the ionic currents flowing through the somatic membrane, it then follows that $V_{\text {ext, } S} \propto \frac{d V_{\text {int, } S}}{d t}$.

If the assumption that $I_{\text {ext }, S} \gg I_{\text {ion, } S}$ is violated, there is no reason to expect that the somatic extracellular potential waveform will be similar to the temporal derivative of the somatic intracellular membrane potential. Therefore, we assume a soma with no AP-generating currents in Figure $4 A$. 
$\mathrm{Na}^{+}$channel density

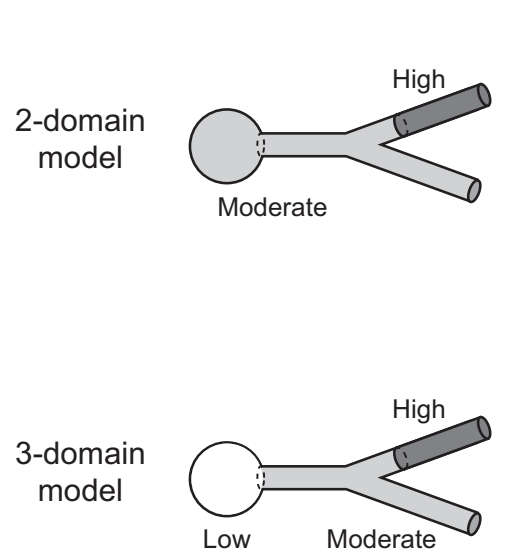

Derivative of somatic intracellular potential
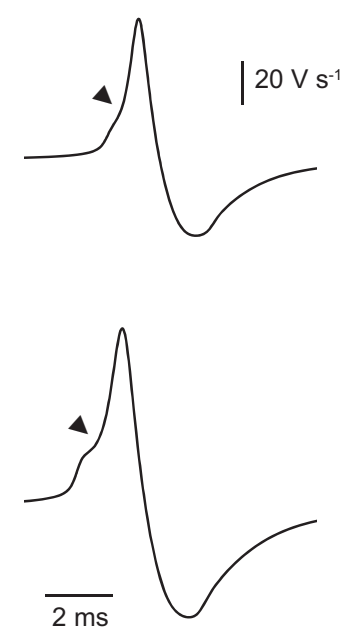

Somatic extracellular potential
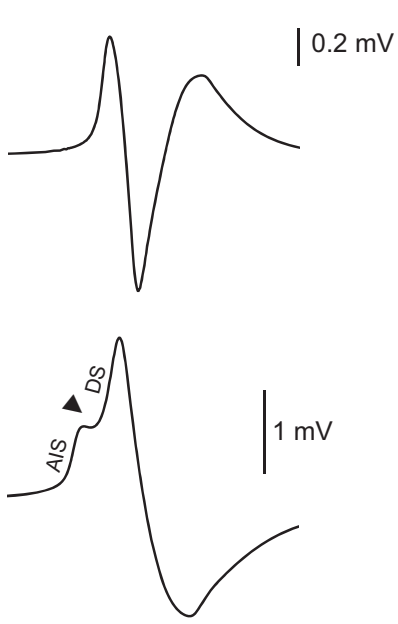

Figure 5. A three-domain model with lower somatic sodium channel density gives rise to a two-component waveform and a notch on extracellular AP. The AIS length was $75 \mu \mathrm{m}$. In the two-domain model (top row), there is no conductance difference between soma and dendrites and corresponds to the model used for all previous figures (left). Although the first derivative of intracellular somatic membrane potential has a notch (arrowhead) in the rising phase, the extracellular AP does not. In addition, the somatic extracellular AP is triphasic. In the three-domain model (bottom row), the somatic $\mathrm{Na}^{+}$and $\mathrm{K}^{+}$conductances were decreased by a factor of 4 and 8.4, respectively, compared with the dendrites. In this case, the extracellular AP does resemble the first derivative of intracellular AP, including the appearance of a notch (arrowheads).

The conceptual model in Figure 4 postulates the following sequence of events to produce a two-component EAP (with its notch) when recorded adjacent to soma. Pacemaking mechanisms in a DA neuron bring the AIS to spike threshold first because of its lower threshold, allowing positively charged $\mathrm{Na}^{+}$ions to enter the AIS via $I_{i o n, A}$, which makes the AIS a sink, and the other domains, sources (Fig. 4A1). This would correspond to a positive deflection in the somatic EAP associated with the AIS component. The axial spread of depolarization then initiates a spike in the ABD portion, which implies that the ABD becomes the dominant sink due to the entry of $\mathrm{Na}^{+}$ions that chiefly comprise $I_{i o n, D}$ (Fig. 4A2). This is manifested as a further positive deflection in the somatic EAP, this time due to the ABD spike. Both events generate large axial currents $\left(I_{\text {axial, AD }}, I_{\text {axial,DS }}\right)$ that cause the soma to be a source as the circuit is completed in the extracellular space. The AP in the AIS repolarizes before the DS spike; because the AIS is more hyperpolarized, the soma becomes a sink during AP repolarization (Fig. 4A3), which manifests as a negative deflection in the EAP. During these events, the somatic membrane current is largely capacitive $\left(I_{C a p, S}\right)$ and the somatic membrane potential is essentially a passive follower of the potential in adjacent compartments. These results suggest that, in order for a DA neuron model to reproduce somatic EAP with an AIS component and a notch in the rising phase, the model must possess three different functional domains: two spike generating domains with different thresholds, and a passive or weakly active third domain (here assumed to be the soma; see Discussion), which is not able to generate spikes on its own.

\section{Simulating the two-component extracellular AP}

The previous section suggested a model manipulation that would allow it to reproduce the observed two-component extracellular waveform with a notch, namely, reducing the contribution of ionic somatic membrane currents during the AP. Somatic $\mathrm{Na}^{+}$ and $\mathrm{K}^{+}$channel conductance densities were decreased by a factor of 4 and 8.4, respectively (Table 2). Consequently, this version of the model has three parametric domains with distinct conduc- tance densities: the AIS, the ABD plus the other dendrites, and the soma. Although the other dendrites have the same parameters as the $\mathrm{ABD}$, they are not considered part of any of the three functional domains in Figure 4 because, in the model, they happen after the somatic AP and do not contribute substantially to the somatic EAP.

In the three-domain model, using the actual value for AIS length $(42 \mu \mathrm{m})$ did not produce a notch, consistent with the EAP actually recorded from this neuron (Fig. 1A, left). Although larger AIS lengths were not able to produce a notch in the EAP (Fig. 3B, top) with only two domains in the model, larger AIS lengths did produce a clear notch in the intracellular first derivatives (Fig. $2 B, \mathrm{dVm} / \mathrm{dt}$, middle row, red trace). Therefore, the AIS length was artificially increased to $75 \mu \mathrm{m}$. With the $\mathrm{Na}^{+}$ channel density in the soma reduced steeply and a larger AIS, the AIS component and the notch in the EAP became visible. The highest density that met this constraint was $200 \mu \mathrm{S} / \mathrm{cm}^{2}$ for $\mathrm{Na}^{+}$ channels for soma (with $800 \mu \mathrm{S} / \mathrm{cm}^{2}$ for ABD and $4000 \mu \mathrm{S} / \mathrm{cm}^{2}$ for AIS). This produced an EAP waveform that resembles the derivative of the somatic intracellular potential, including the notch in the rising phase (Fig. 5, three-domain model). Also importantly, the triphasic AP in the two-domain model was replaced by the biphasic AP that is observed in unfiltered extracellular recordings (Ungless and Grace, 2012).

Figure 6 repeats the simulations on the effect of morphological manipulations on the intracellular AP waveform with the reduced somatic conductance (three-domain) model, which were previously performed using the initial and more parsimonious two-domain model (Fig. 2). We found that the observed effects of AIS and ABD morphology on intracellular AP waveform and its temporal derivatives are maintained when recording from a "passive" soma in the three-domain model, thus highlighting the point that very similar intracellular AP waveforms can produce dramatically different extracellular counterparts.

To more diagrammatically and intuitively illustrate how the reduced active currents in the soma alter the local sources and sinks that determine the EAPs in the two models, we simulated 
A

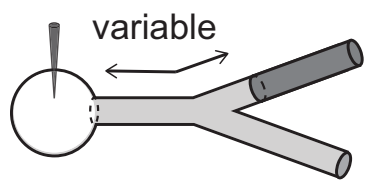

distance to soma $(\mu \mathrm{m})$
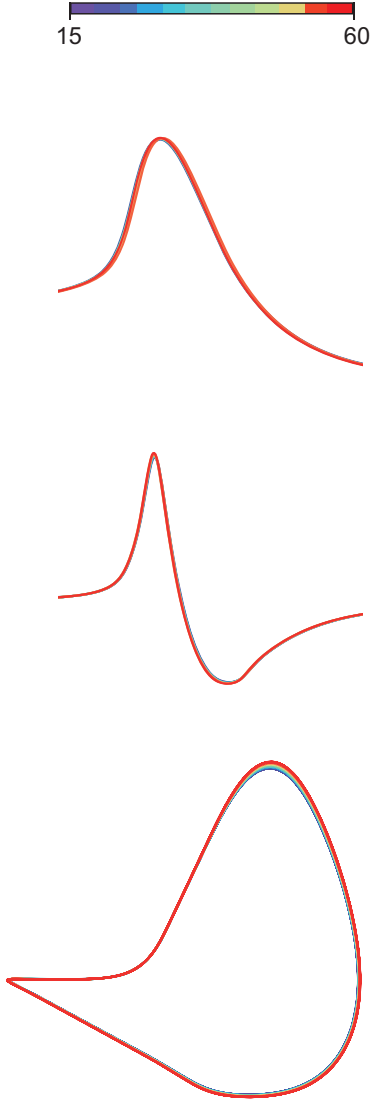

B

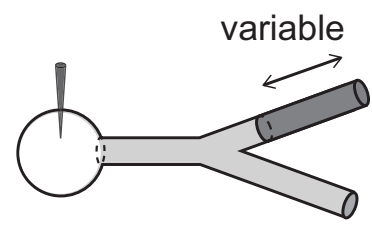

length AIS $(\mu \mathrm{m})$
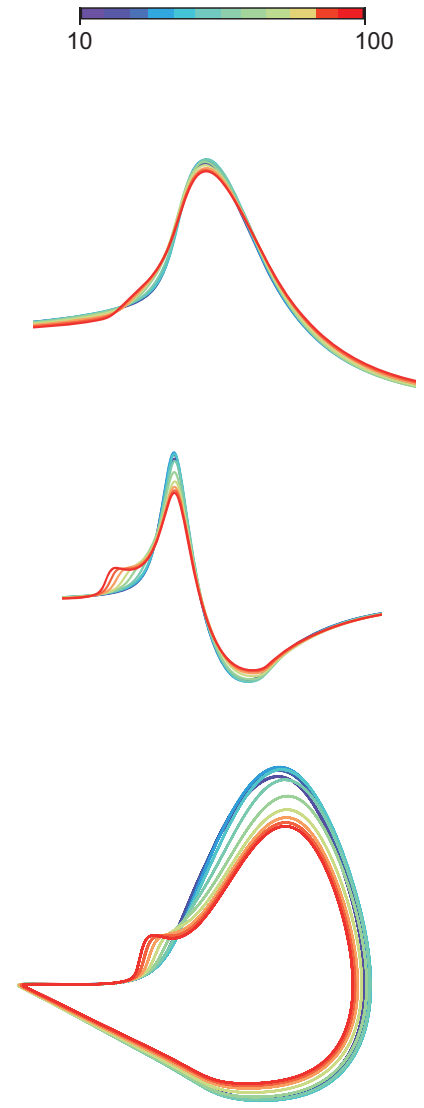

C
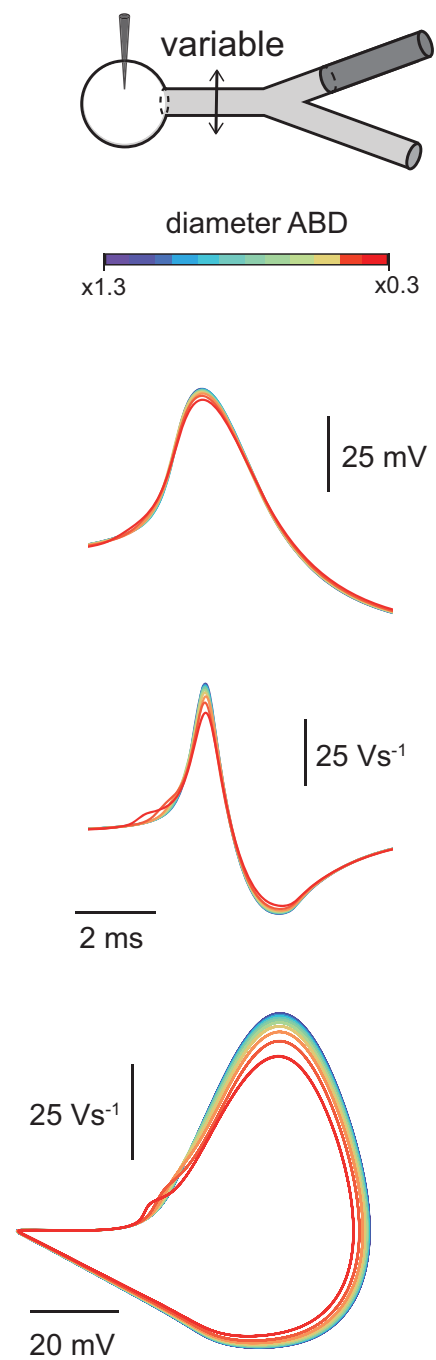

Figure 6. Predicted influence of AIS distance, AIS size, and ABD diameter on somatic intracellular AP shape in the three-domain model. A, Varying the distance from soma to proximal border of AIS (equivalent to varying in ABD length, top and color code bar) from 15 to $60 \mu \mathrm{m}$ in increments of 5 does not affect intracellular AP shape (top), first temporal derivative (middle), or the phase plane plot (bottom) recorded in the soma (top). AIS length held constant at its true value of $41.57 \mu \mathrm{m}$. ABD diameter tapers from 4.25 to 3.05 to 0.83 as in original reconstruction. $\boldsymbol{B}$, Increasing AIS length (top and color code bar) from 10 to $100 \mu \mathrm{m}$ in increments of 10 makes the kink more prominent in the intracellular potential (top, arrow). It also makes the AIS component and the notch that follows it more prominent in the first temporal derivative and phase plane plots (middle, bottom). Als distance held constant at $29.36 \mu \mathrm{m}$. ABD diameter tapers from 4.25 to 3.05 to 0.83 as in original reconstruction. C, Decreasing ABD diameter (top and color code bar) by a factor of 1.3 to 0.3 in decrements of 0.1 makes the kink more prominent in the intracellular AP (top). Decreasing ABD diameter also makes the AIS component and notch more visible. AIS distance held constant at $29.36 \mu \mathrm{m}$ and AIS length held constant at $41.57 \mu \mathrm{m}$.

extracellular electrodes near the AIS (red traces), near the ABD (blue traces), and near the soma (black traces), along with the simultaneous intracellular recording (Fig. 7). As in Figure 5, we used the model neuron with the AIS length artificially increased to $75 \mu \mathrm{m}$ in order for this particular morphology to exhibit a notch in the somatic EAP. As seen in the first row of Figure 7, the intracellular recorded APs do not differ greatly between models: in both, the AIS fires first, then the ABD and soma fire simultaneously, as shown earlier (Fig. 1D).

Also, in both models, the EAP near the AIS (Fig. 7, second row, red traces) is a biphasic negative-positive signal, demonstrating that the spike initiates at the AIS and then spreads to the adjacent $\mathrm{ABD}$ and soma. The observed first negative deflection in the EAP signifies that the underlying intracellular compartment (in this case the AIS) is a sink for positive charge relative to its neighboring compartments. The subsequent positive deflection signals that the AIS compartment has become a source as it repolarizes, and as the ABD fires and becomes a sink.

The EAP near the ABD is also similar in both models (Fig. 7, third row, blue traces). In both, it is a triphasic AP, starting with a positive phase before the negative-positive signal. The observed first positive deflection reflects that the ABD is first a source for the AIS spike. The subsequent negative deflection reflects that the $\mathrm{ABD}$ is then a sink during the active phase of the ABD spike. Finally, the ABD becomes a source again as it repolarizes.

The crucial difference between models lies in the somatic EAP (Fig. 7, fourth row, black traces), as also previously shown (Fig. 5). In the two-domain model (left side), the somatic EAP is triphasic and does not exhibit a notch. Because in this model the soma is active, it generates a spike simultaneously with the ABD (DS spike). Thus, the observed first positive deflection (fourth row, left side) reflects that the soma becomes a source for the AIS 
A1
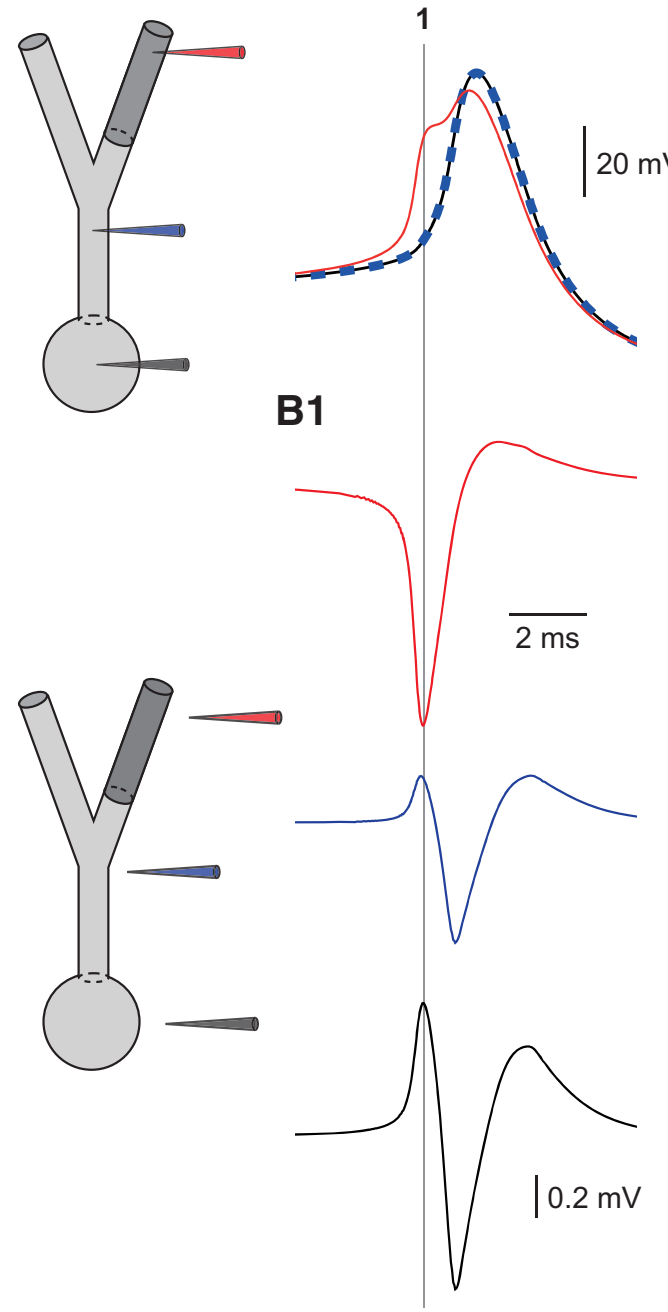

A2

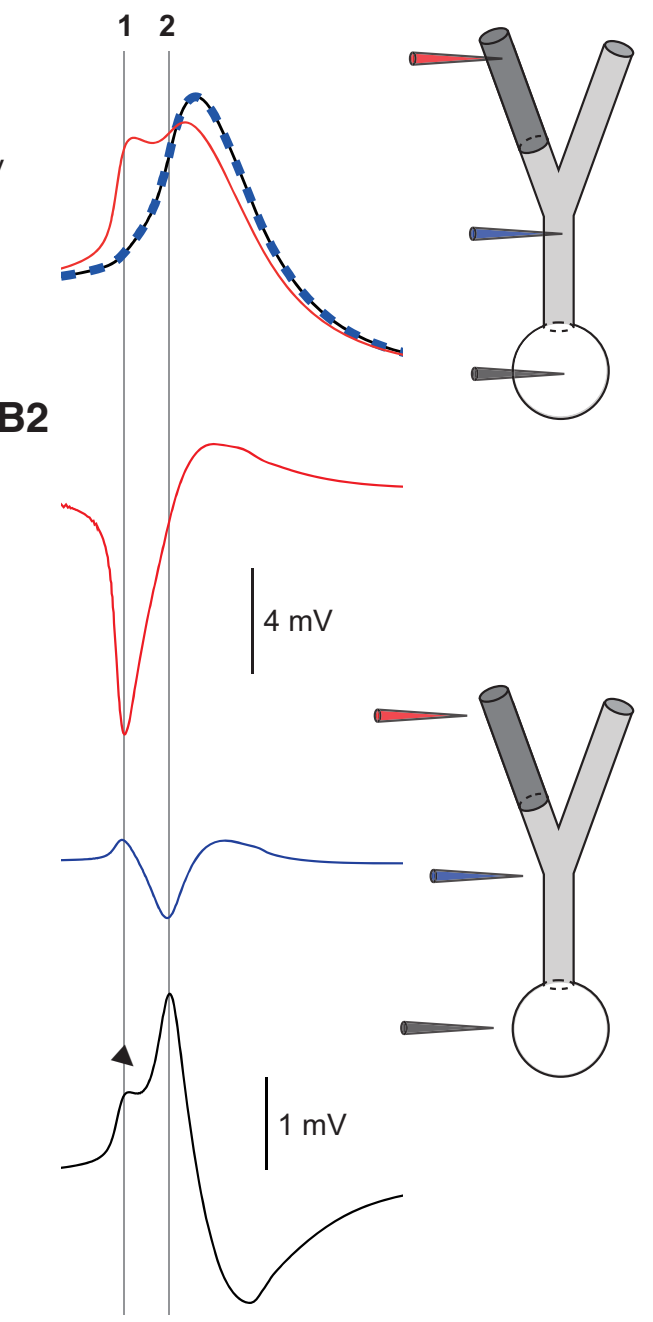

Figure 7. Spatiotemporal relationship between extracellularly and intracellularly simulated APs. Simultaneous intracellular and extracellular AP traces are shown for different locations using a two-domain (left) and a three-domain (right) model. The AlS length was setto $75 \mu \mathrm{m}$. The adjacent diagrams indicate the simulated placement of electrodes: inside (A1, $\boldsymbol{A 2}$, top) and outside (B1, B2, bottom) of soma (black), $A B D$ (blue), and AIS (red) compartments. Intracellular traces were superposed for the three compartments, whereas extracellular traces are positioned according to their relative locations (Fig. $4 A$ ). In both left and right columns, the vertical line (1) indicates the time when the negative peak of AIS extracellular spike occurs. In the right column, the second line (2) indicates the positive peak of the extracellular somatic spike. Intracellular waveforms in the three compartments are similar between models. Extracellular AP for electrodes located at the AIS and ABD are also similar between models. The AIS APs (red traces) are negative-positive-biphasic waveforms, reflecting the AIS is a sink when firing, and then a source when repolarizing. The ABD APs (blue traces) are triphasic positive-negative-positive, reflecting that the ABD is first a source for the AIS spike, then a sink for the ABD spike, and then a source when repolarizing. Somatic APs are different between models. In the two-domain model, the somatic AP is a positive-negativepositive triphasic waveform reflecting that the soma is first a source for the AIS spike, then a sink for the somatic spike (which happens simultaneous with the ABD spike, see intracellular traces), and then a source when repolarizing. In the three-domain model, the soma does not generate an AP, passively following the ABD spike. This results in a positive-negative biphasic waveform with a notch, reflecting the soma is first a source for the AIS spike, then a source again for the ABD spike, and then a sink when repolarizing (Fig. 4A).

spike, and the subsequent negative deflection (fourth row, left side) reflects that the soma becomes a sink during the active phase of the simultaneous DS spike. Finally, the subsequent positive deflection indicates that the soma (together with the $\mathrm{ABD}$ ) becomes a source again as it repolarizes.

In the three-domain model (right side), on the other hand, the somatic EAP is biphasic and presents a clear notch (fourth row, black traces). The first positive deflection (fourth row, right side) reflects that the soma is a source for the AIS spike. Yet, because in this model the soma is passive or weakly active, it will not generate a spike on its own. This implies that, instead of becoming a sink together and simultaneously with the ABD spike, the soma will be instead a source for the ABD spike. This will produce the second positive deflection in the somatic EAP, resulting in a notch (fourth row, right side). The fact that the soma does not generate a spike on its own does not impede its depolarization when the ABD generates a spike. Therefore, the observed somatic notch (arrowhead) can be explained as the transition from being a source for the AIS sink to being a source for the ABD sink. Finally, the negative deflection reflects the repolarization of the AIS and of the ABD, which the soma follows passively. This proposition is consistent with the observation that the EAP in midbrain dopamine neurons is characteristic of two distally generated spikes, which have the soma as their current source (Grace, 1990).

\section{AIS and ABD morphology affects the amplitude and timing of the AIS component and notch}

We examined the role of morphology in the prominence of notch in the EAP in the three-domain model. The distance from cell body to proximal AIS border had a small effect on the promi- 
A

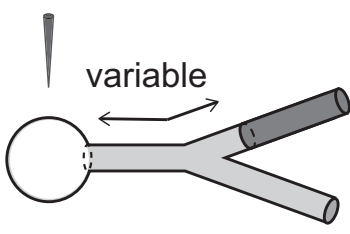

distance to soma $(\mu \mathrm{m})$

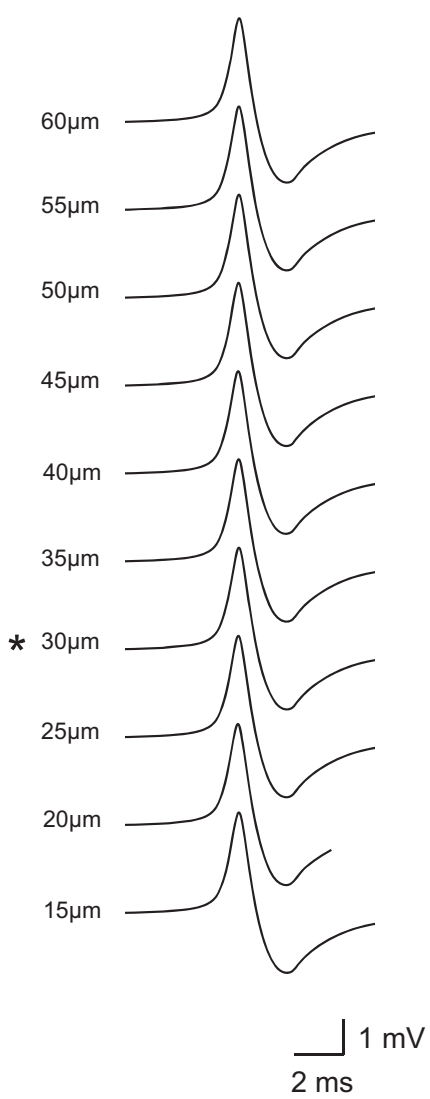

B

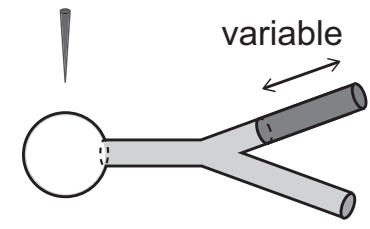

length AIS $(\mu \mathrm{m})$

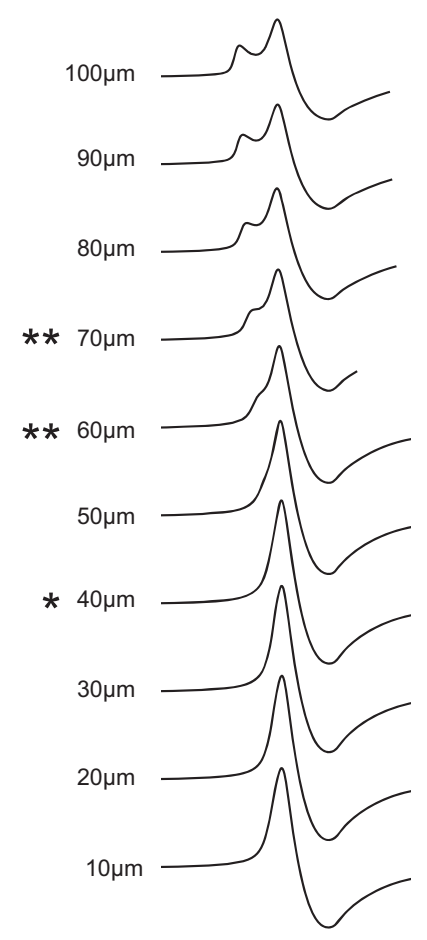

C

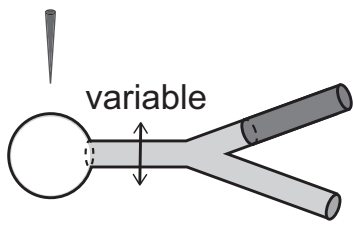

diameter $\mathrm{ABD}$

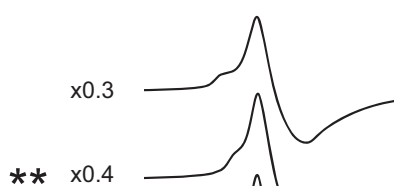

** $\times 0.4 \longrightarrow$

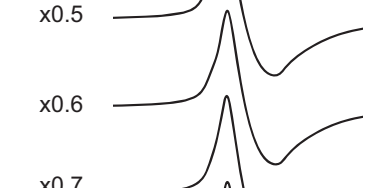

$\mathrm{x} 0.7$

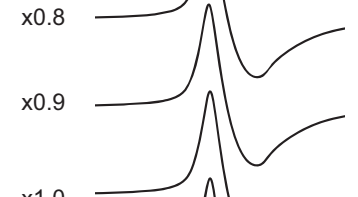

*

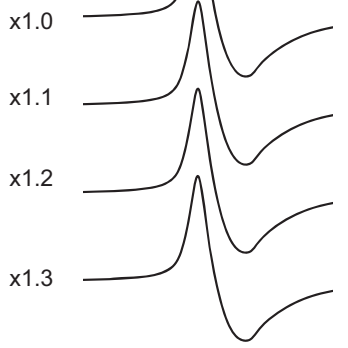

Figure 8. AIS length strongly influences the AIS component and notch in extracellular AP shape in the three-domain model. $\boldsymbol{A}$, Increasing the distance from the soma to the AIS proximal end (equivalent to increasing ABD length, top) does not affect the prominence of the AIS component. $\boldsymbol{B}$, Increasing AIS length (top), on the other hand, increases amplitude of the AIS component (top). Decreasing the AIS length renders the AIS component and notch invisible (bottom). C, Decreasing ABD diameter (top) produces a temporal separation between the AIS component and the main spike (top). Increasing the diameter to larger values renders the AIS component and notch less evident (bottom). *The original morphological value. **The shape of what is typically observed in extracellular recordings of DA neurons.

nence of the AIS component or the notch (Fig. 8A), with longer distances slightly decreasing the magnitude of the AIS component. On the other hand, increasing AIS length dramatically increased the amplitude of the AIS component and the notch; only the smallest AISs failed to produce a clear AIS component and notch (Fig. $8 B$ ). We observed similar results when the size of the AIS was increased by increasing the AIS diameter, an expected result given that the surface area controls the magnitude of the AIS current (data not shown). Decreasing the ABD diameter also resulted in a notch, but only with the smallest diameters (Fig. $8 \mathrm{C}$ ), which is consistent with what was observed in the first temporal derivative and phase plane plots (Figs. 2, 6). These results show that the effect of AIS size on the intracellular (Fig. 6) and extracellular somatic APs is consistent, and predict that AIS component and notch in the somatically recorded EAP will be more prominent in neurons with larger AIS and, although to a lesser extent, thinner ABD.

\section{Convergence of two ways to calculate the EAP}

We used the NEURON extracellular mechanism xtra to calculate the EAPs in Figures 3, 5, 7, and 8; the LFPy software (Lindén et al., 2014) takes a different approach (see Materials and Methods) but produces similar results (Fig. 9), which increases our confidence in the validity of our results. Figure 9 shows normalized, simulated EAPs at various locations around the simulated threedomain mouse neuron for two AIS lengths: the actual length 42 $\mu \mathrm{m}$ in Figure $9 A$ and an artificially lengthened AIS $(75 \mu \mathrm{m})$ to illustrate the emergence of a notch. Figure $9 A 1$ shows that similar biphasic waveforms without a notch are predicted near the soma and primary dendrites, consistent with our experimental observations. Figure $9 A 2$ and Figure 9A3, respectively, show an EAP recorded near the soma and one recorded near the AIS. In agreement with the data in Figure $1 A$ (left), there is no notch in Figure $9 A 2$. Figure $9 B$ shows the same simulations for a model neuron with an artificially lengthened AIS $(75 \mu \mathrm{m})$. The shapes in Figure 
$9 B 2, B 3$ are qualitatively similar to the corresponding EAPs in Figure $7 B$. As expected from the theory in Figure $4 A$, and model results in Figure $7 B$, the AIS component and notches are only observed in simulated recordings near the soma (Fig. 7B2). In general, experimentalists look for the largest amplitude waveform when recording a single unit: the largest amplitude simulated waveforms are the red, orange, and yellow waveforms in that order. They are all near the soma and exhibit a physiologically plausible notch. We also confirmed the presence of a triphasic AP by placing the simulated electrode near the ABD (corresponding extracellular traces in Figs. 7, 10; and data not shown). Consistent with theory, there is a presence of triphasic APs in very distal dendrites (Fig. 9B, second to fourth EAPs of last row); but based on how experimental EAPs are recorded, these smallamplitude waveforms are not likely to be observed in practice.

The effects of changing the AIS length, AIS distance to the soma, and diameter were consistent across parameter sets, including an earlier set (data not shown) with higher sodium $\left(70,000 \mu \mathrm{S} / \mathrm{cm}^{2}\right)$ and delayed rectifier $\left(18,000 \mu \mathrm{S} / \mathrm{cm}^{2}\right)$ conductance densities and lower $\mathrm{R}_{\mathrm{a}}(30$ $\Omega-\mathrm{cm})$.

\section{Replication of the model on a neuron with a physiological two-component EAP}

The work in this study was motivated by and driven by our database on the morphology of mouse SNc DA neurons in which the size and location of the AIS and the 3D structure of all compartments are precisely known (Meza et al., 2018). Although our study showed what morphological changes (specifically a larger AIS) would be required to produce a notch in the EAP, we did not observe a notch in that population experimentally (Meza et al., 2018). Therefore, we simulated the electrical activity of a rat neuron that did display a physiological EAP with the notch (Fig. 1A, right) and whose morphology is also known precisely (Fig. 10A). AIS length data was not available for this neuron, so the length of its AIS was estimated (see Materials and Methods) at $37 \mu \mathrm{m}$. The same parameters as given in Tables 2 and 3 were applied to a model implemented in the reconstructed morphology for this neuron. Without any adjustments, the model exhibited spontaneous pacemaking activity (Fig. 10B, inset). The AP initiated in the AIS as before (Fig. $10 B)$. Remarkably, the two- and three-
A1

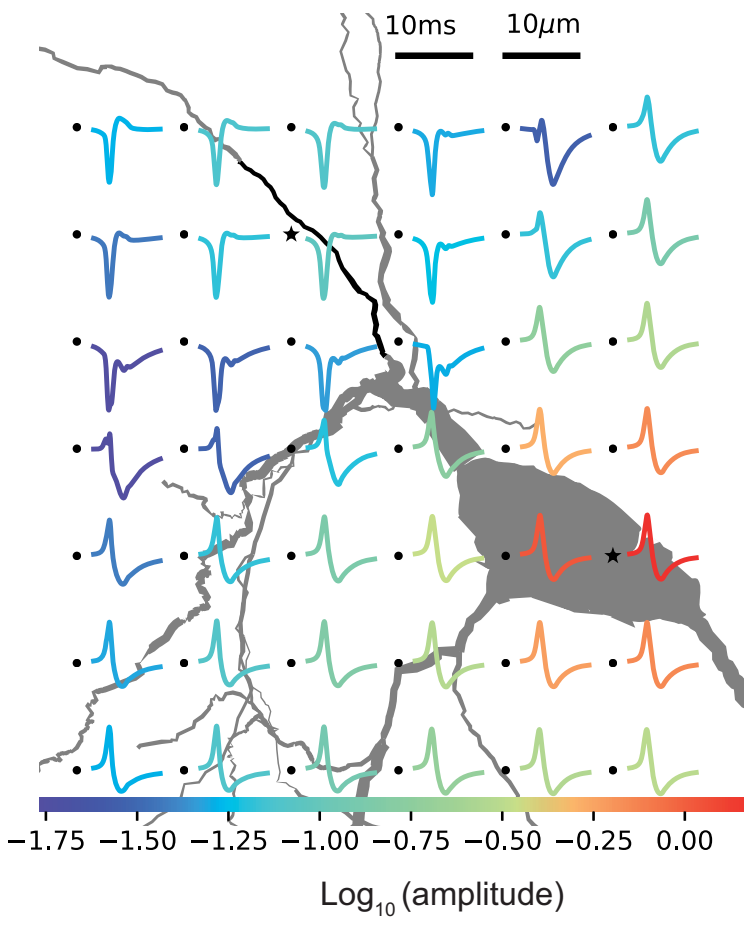

B1

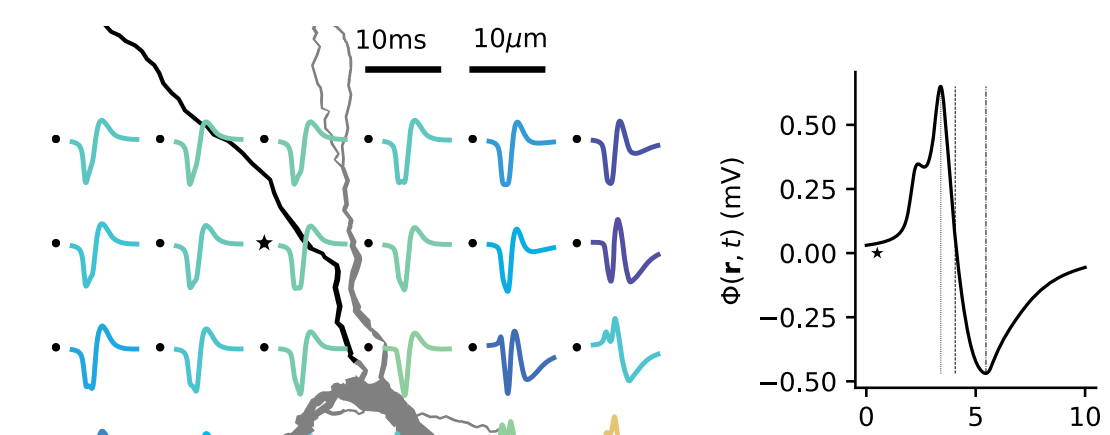

A2

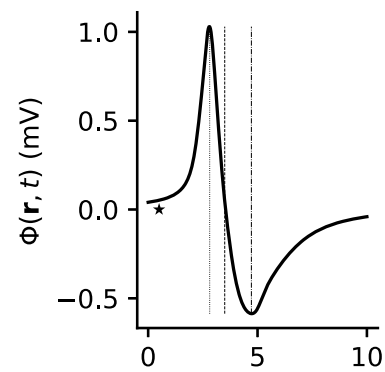

A3

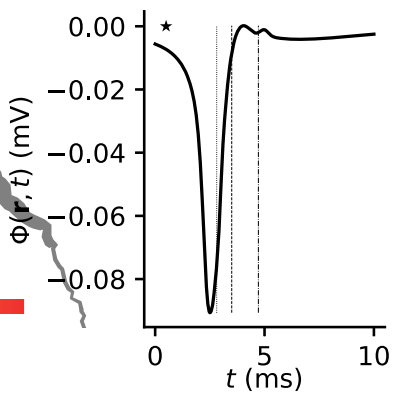

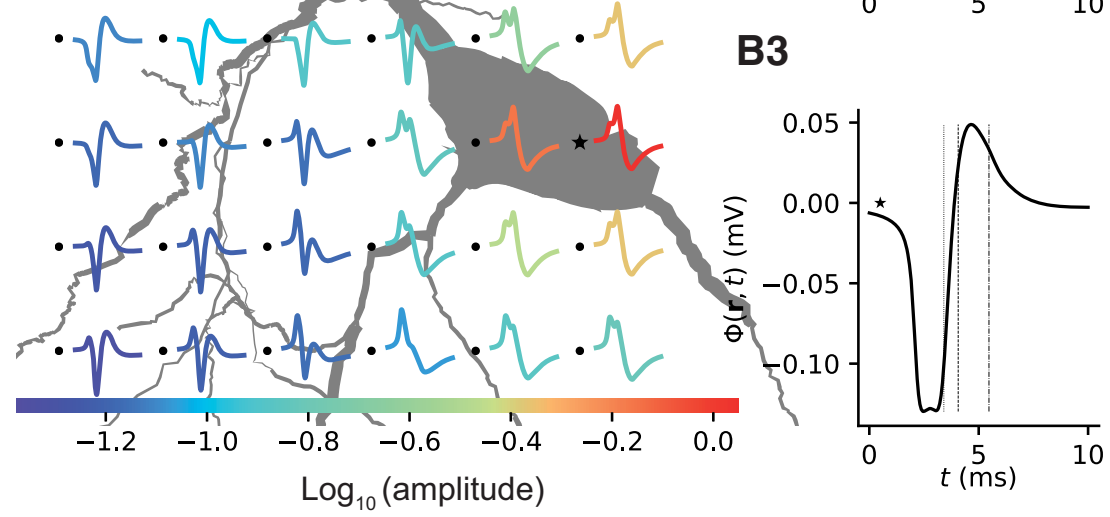

Figure 9. Calculated extracellular spike waveforms for mouse neuron using LFPy. A1, Position-dependent extracellular spike waveforms during an AP. Black dots indicate the positions of the (virtual) electrode contact points. The largest EAP occurs near the soma (red trace). $\boldsymbol{A}$ 2, Extracellular waveform during AP at the position denoted with the bottom asterisk in $\boldsymbol{A} \mathbf{1}$ (near the soma). $\boldsymbol{A}$, Extracellular waveform during AP is shown at the position denoted with the top asterisk in $\boldsymbol{A} \mathbf{1}$ (near the AIS). $\mathbf{A 2}, \mathbf{A} \mathbf{3}$, Vertical dashed lines indicate temporal alignment with the minimum negative extracellular peak in the close-to-AIS electrode, the maximum magnitude of intracellular AP, and the maximum of positive extracellular peak in the close-to-AIS electrode. $\boldsymbol{B}$, The AIS length was artificially increased to $75 \mu \mathrm{m}$ as in Figures 5 and 7 to produce a notch; $\mathbf{B 1}, \mathbf{B 2}$ and $\mathbf{B} 3$ contain the same information as in $A$ for the modified morphology. The AIS component and notches are only observed in recordings near the soma (yellow, orange, and red traces). There is a presence of triphasic AP in distal dendrites (first to third EAP in bottom two rows of $\boldsymbol{B}$ ). The spike amplitude is the voltage difference between the minimum and the maximum. All waveforms are normalized to have the same distance between the negative and positive peaks, and the magnitude of this distance is given by the logarithmically scaled color code. 
A

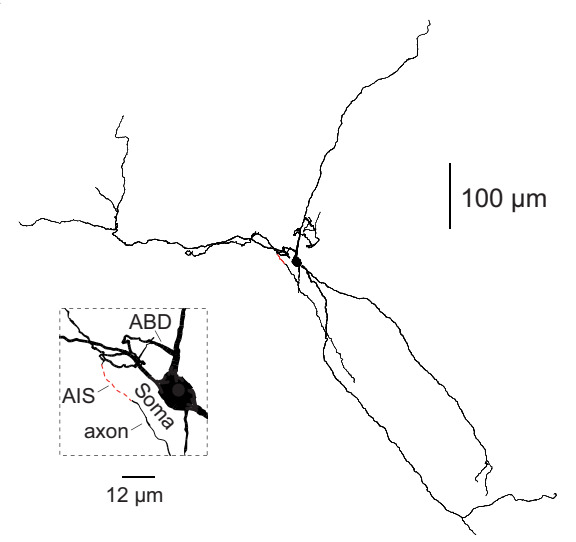

C

$\mathrm{Na}^{+}$channel density

3-domain model
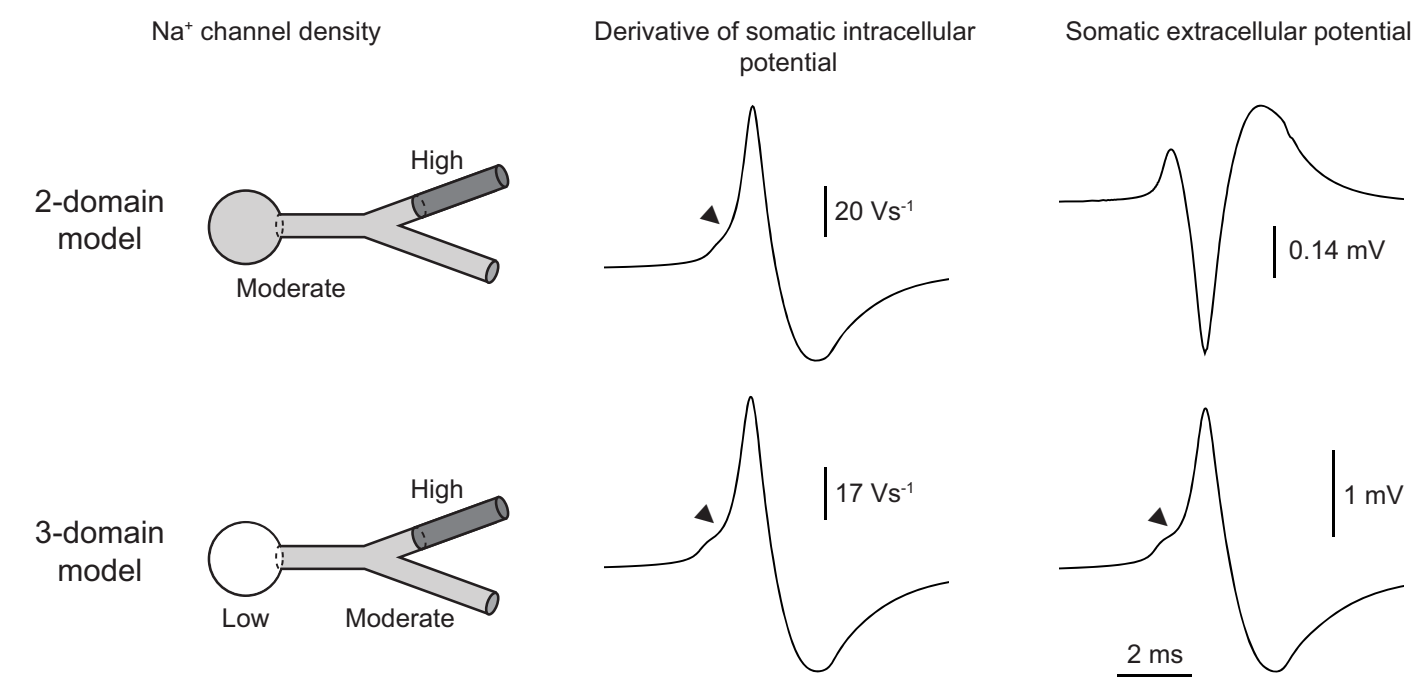

B

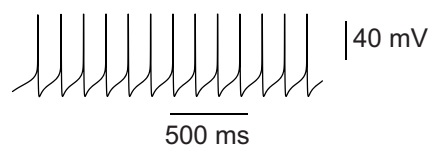

$20 \mathrm{mV}$

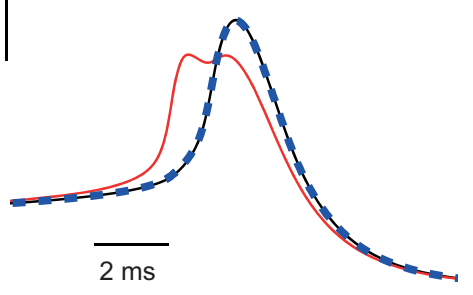

Figure 10. Model parameter settings generalize to a neuron with a physiologically recorded EAP notch. $A$, Morphology of a rat SNc DA neuron that exhibits a notch (Fig. $1 A$, right). Black represents somatic, dendritic, and axonal compartments. Red represents AIS. Left, Inset, Magnified view of the precise architectural arrangement to indicate the experimentally determined AIS location and the estimated AIS length (dashed red line). B, Simulated pacemaking (top, inset) and intracellular waveforms for the AIS (red), ABD (blue dashed), and soma (black). C, Without any parameter changes, the rat neuron model recapitulates the results in the mouse neuron model and correctly produces a notch in the derivative intracellular waveform (middle, arrowheads). As in the mouse neuron, the somatic sodium conductance density had to be reduced to observe the notch in the extracellularly recorded AP (rightmost, arrowhead), which should be compared with the rightmost trace in Figure $1 A$.

domain models recapitulated the results from the mouse neuron. Both models captured the notch (arrowheads) in the derivative of the intracellular waveform (Fig. 10C, middle), which is expected given the notch observed in the EAP in Figure 1. Moreover, the two-domain model once again produced an EAP without a notch, whereas the three-domain model correctly produced it (arrowhead) in Figure 10C (right). The correct production of a two-component EAP and a notch in the second neuron model is all the more remarkable because the estimated AIS in the rat neuron model that produced a notch was actually smaller than the one in the mouse neuron model that did not produce a notch. This indicates that other aspects of the morphology are also important, and the relationship between these other aspects and the AIS determines whether a notch is observed rather than just the size of the AIS alone. For example, the maximum ABD diameter in the rat model is $1.55 \mu \mathrm{m}$, approximately one-third of the maximum diameter in the mouse model $(4.25 \mu \mathrm{m})$, consistent with the finding in Figure $8 C$ that smaller ABD diameter on the EAP waveform can enhance the notch. Also, the rat neuron has only a $56 \%$ of the combined somatodendritic surface area compared with the mouse neuron (Table 1), which could accentuate the contribution of the AIS.

\section{Discussion}

We show that the somatically recorded two-component EAP, which is visible as a notch, of SNc DA neurons requires the presence of three domains with distinct excitability characteristics (three-domain model). Given the presence of three domains, we demonstrated that variability in AIS size, and to a lesser extent ABD diameter, affects the amplitude of the AIS component of the EAP and notch. In contrast, AIS-to-soma distance has little effect. Strikingly, the model correctly reproduced the notch in a neuron with a different morphology than the one for which the model was calibrated. Finally, this study shows EAP waveform analysis provides information on the biophysical properties of neurons that are inaccessible from intracellular waveform analysis alone.

\section{Computational model of the extracellular AP of DA neurons}

This is the first attempt to systematically model the EAP waveform of DA neurons. The notch of EAP is frequently reported in 
extracellular studies (Grace and Bunney, 1983a, b; Brown et al., 2009; Henny et al., 2012; Ungless and Grace, 2012), and sometimes used as an electrophysiological criterion for identification of DA neurons (Tepper et al., 1995; Drion et al., 2010). Here we show that to observe the notch in the somatic EAP, two domains must generate active spikes, but with different thresholds such that one precedes the other (Figs. 1, 7, 10), consistent with an AIS/DS dipole (Telenczuk et al., 2018). The AIS domain must have the lowest threshold (Gentet and Williams, 2007). We hypothesize that the second domain is the $\mathrm{ABD}$, and predict that its contribution to the somatic EAP is more prominent than that from other equally active dendrites because of its privileged access to the AIS and proximity to the soma such that it directly triggers the somatic depolarization. A third domain, most likely the soma, should be the major contributor to the recorded EAP, and be passive during the AP, meaning that the capacitive current is mostly comprised of axial current from other domains, with a minimal contribution of intrinsic ionic membrane currents. These conditions are consistent with earlier conceptual models and required by circuit theory (Fig. 4) for the extracellular waveform to resemble the time derivative of intracellular potential (Hodgkin and Huxley, 1952).

\section{Information provided by the extracellularly recorded AP} The EAP waveform provides information about the cell's morphological and biophysical characteristics that cannot be extracted from the intracellular AP (Gold et al., 2006). In this work, we show that analyzing the intracellular or first temporal derivative waveform analysis is insufficient to reveal the requirement of a weakly active soma for a two-component EAP (or notch) (compare Fig. $2 B$ with Fig. $3 B$ ). In addition, the EAP waveform provides stronger clues about AIS and ABD morphology (Fig. 8). Finally, we found that reproducing the shape of recorded EAP also resulted in a more tightly constrained model. As mentioned in Materials and Methods, a nonphysiological notch on the EAP negative phase was revealed during the development of the EAP model. The introduced changes in the time constants strongly impacted the EAP with little effect on the intracellular AP. In practice, this resulted in a better model of the $\mathrm{Na}^{+}$channel kinetics than could be obtained by simply mimicking the intracellular AP.

\section{Correspondence between extracellularly and intracellularly recorded AP}

The semiquantitative theory presented in the text accompanying Figure $4 A$, which shows a correspondence between the EAP recorded near the soma and the first temporal derivative of the somatic intracellular potential, makes restrictive assumptions. The EAP is actually due to the sum of the ionic currents and the capacitive current (Hodgkin and Huxley, 1952); but as shown in Figure 4, only the capacitive current is proportional to the intracellular first derivative. For a two-compartment model with a passive compartment, the approximation is good (Freygang and Frank, 1959). However, as the morphological complexity is increased by adding more compartments, the relationship between the two waveforms becomes more complicated, as observed in real datasets (Rall and Shepherd, 1968; Holt and Koch, 1999; Henze et al., 2000). For example, in CA1 pyramidal neurons, these waveforms were similar during the ascending phase of the intracellular AP but differed substantially during the descending phase and spike afterhyperpolarization (Henze et al., 2000).

\section{Comparison with the Grace and Bunney earlier model}

Grace and Bunney (1983a) proposed a conceptual model to explain the AIS and somatodendritic components of the recorded EAP. During autonomous pacemaking, the soma depolarizes the neuron during the interspike interval. The AIS reaches threshold first, initiating the AIS spike, and becoming a sink (source) with respect to the extracellular (intracellular) space. In contrast to the original paper (Grace and Bunney, 1983a), we use the extracellular convention, so during the AIS spike, the soma is a source. Next, another domain with a higher threshold (suggested to be distal dendrites on the opposite [contralateral] side on the soma from the AIS) fires, causing the soma to remain a source during what they termed the somatodendritic phase. It is implicit in their conceptual model that the axial current from both the AIS and distal dendrite spikes dominates the somatic transmembrane current, and that the soma does not generate an AP but rather passively follows the other domains. Our model is conceptually quite similar, only differing in the locus of the somatodendritic spike: a distal dendrite in their model versus the ABD in ours. APs are first recorded from the ABD near the AIS, then spread to the soma and only then to the contralateral dendrites (Häusser et al., 1995; Gentet and Williams, 2007). Based on this experimental evidence, in our model, the sequence of events is as follows: occurrence of the AIS spike, induction of a regenerative AP that travels down the axon and backpropagates to the $\mathrm{ABD}$ and the remainder of the ipsilateral dendritic arbor, occurrence of the $\mathrm{ABD}$ spike, depolarization of the soma, and induction of spikes in the remaining contralateral dendritic arbor.

\section{Passive versus active soma in DA neurons}

Evidence supports a passive soma in spinal sensory and motor neurons (Safronov et al., 2000) and in cochlear nucleus auditory neurons (Yang et al., 2016). However, the only comparative study (Häusser et al., 1995) in rat nigral DA neurons found no significant difference in $\mathrm{Na}^{+}$channel density between soma and the active dendrites. Yet, they did observe a greater TTX-dependent attenuation of the AP in the dendrites compared with the soma, suggesting a greater influence of $\mathrm{Na}^{+}$channels in the dendrites. Also, the soma of DA neurons is at least less active than that of substantia nigra reticulata neurons (Seutin and Engel, 2010). On the other hand, the soma of DA neurons is not entirely passive; at a minimum, it participates in the slow depolarizations between APs that drive pacemaking (Grace and Bunney, 1983a). Further studies comparing somatic and $\mathrm{ABD} \mathrm{Na}{ }^{+}$channel density in a variety of identified DA neurons could clarify this issue. Still, it is theoretically plausible that inhibition of the somatic compartment could contribute to lower net somatic ionic currents.

Less than half of neurochemically identified rat nigral DA neurons exhibit a notch (Brown et al., 2009) in the EAP. No notch was reported in neurochemically (Meza et al., 2018) or genetically (Lerner et al., 2015) identified mouse nigral DA neurons, but notches were reported in identified mouse ventral tegmental area DA neurons (Schweimer et al., 2014). Variability in AIS (González-Cabrera et al., 2017; Meza et al., 2018), ABD morphology (Häusser et al., 1995; Blythe et al., 2009; Meza et al., 2018), or other biophysical properties across midbrain DA neurons may account for the observed diversity in EAP waveforms.

\section{Implication and predictions of diversity in AIS and ABD morphology}

A model prediction is that AIS size should correlate with the magnitude of the notch in the AP. On the other hand, the distance between the soma and AIS did not have an effect on somatic EAP 
waveform within the range of distances experimentally observed. Similarly, AIS size, but not position, correlated with the in vivo spontaneous firing rate in DA neurons (Meza et al., 2018). We also found that $\mathrm{ABD}$ diameter influenced the magnitude of the AIS component. This could be partly explained as a result of a longer time separation between components due to slower backpropagation in processes with smaller diameters (Hursh, 1939). The rat model neuron produced a notch with a smaller AIS than required for the mouse neuron model, which indicates that other aspects of morphology, such as the diameter of the ABD or somatodendritic surface area, or biophysical diversity across neurons, may play a role in this difference. Our results reveal that the morphology of AIS and ABD substantially affects the shape of EAP. Understanding how morphology affects the EAP is important for developing better algorithms for spike sorting (Barthó et al., 2004) to analyze massive recordings from large samples of neurons (Jun et al., 2017). Moreover, better understanding of the EAP will help us understand its significant contribution to high frequencies in the LFP and EEG (Telenczuk et al., 2015).

\section{References}

Barthó P, Hirase H, Monconduit L, Zugaro M, Harris KD, Buzsáki G (2004) Characterization of neocortical principal cells and interneurons by network interactions and extracellular features. J Neurophysiol 92:600-608. CrossRef Medline

Bean BP (2007) The action potential in mammalian central neurons. Nat Rev Neurosci 8:451-465. CrossRef Medline

Beckstead MJ, Grandy DK, Wickman K, Williams JT (2004) Vesicular dopamine release elicits an inhibitory postsynaptic current in midbrain dopamine neurons. Neuron 42:939-946. CrossRef Medline

Bender KJ, Trussell LO (2012) The physiology of the axon initial segment. Annu Rev Neurosci 35:249-265. CrossRef Medline

Blythe SN, Wokosin D, Atherton JF, Bevan MD (2009) Cellular mechanisms underlying burst firing in substantia nigra dopamine neurons. J Neurosci 29:15531-15541. CrossRef Medline

Brette R (2013) Sharpness of spike initiation in neurons explained by compartmentalization. PLoS Comput Biol 9:e1003338. CrossRef Medline

Brooks CM, Eccles JC (1947) Electrical investigation of the monosynaptic pathway through the spinal cord. J Neurophysiol 10:251-273. CrossRef Medline

Brown MT, Henny P, Bolam JP, Magill PJ (2009) Activity of neurochemically heterogeneous dopaminergic neurons in the substantia nigra during spontaneous and driven changes in brain state. J Neurosci 29:2915-2925. CrossRef Medline

Buzsáki G (2004) Large-scale recording of neuronal ensembles. Nat Neurosci 7:446-451. CrossRef Medline

Cardozo DL, Bean BP (1995) Voltage-dependent calcium channels in rat midbrain dopamine neurons: modulation by dopamine and GABAB receptors. J Neurophysiol 74:1137-1148. CrossRef Medline

Carnevale NT, Hines ML (2006) The NEURON Book. Cambridge: Cambridge UP.

Csicsvari J, Hirase H, Czurkó A, Mamiya A, Buzsáki G (1999) Oscillatory coupling of hippocampal pyramidal cells and interneurons in the behaving rat. J Neurosci 19:274-287. CrossRef Medline

Ding S, Matta SG, Zhou FM (2011a) Kv3-like potassium channels are required for sustained high-frequency firing in basal ganglia output neurons. J Neurophysiol 105:554-570. CrossRef Medline

Ding S, Wei W, Zhou FM (2011b) Molecular and functional differences in voltage-activated sodium currents between GABA projection neurons and dopamine neurons in the substantia nigra. J Neurophysiol 106:30193034. CrossRef Medline

Drion G, Bonjean M, Waroux O, Scuvée-Moreau J, Liégeois JF, Sejnowski TJ, Sepulchre R, Seutin V (2010) M-type channels selectively control bursting in rat dopaminergic neurons. Eur J Neurosci 31:827-835. CrossRef Medline

Durante P, Cardenas CG, Whittaker JA, Kitai ST, Scroggs RS (2004) Lowthreshold L-type calcium channels in rat dopamine neurons. J Neurophysiol 91:1450-1454. CrossRef Medline

Fatt P (1957) Electric potentials occurring around a neurone during its antidromic activation. J Neurophysiol 20:27-60. CrossRef Medline
Fleidervish IA, Lasser-Ross N, Gutnick MJ, Ross WN (2010) $\mathrm{Na}^{+}$imaging reveals little difference in action potential-evoked $\mathrm{Na}^{+}$influx between axon and soma. Nat Neurosci 13:852-860. CrossRef Medline

Ford CP, Gantz SC, Phillips PE, Williams JT (2010) Control of extracellular dopamine at dendrite and axon terminals. J Neurosci 30:6975-6983. CrossRef Medline

Freygang WH Jr, Frank K (1959) Extracellular potentials from single spinal motoneurons. J Gen Physiol 42:749-760. CrossRef Medline

Gentet LJ, Williams SR (2007) Dopamine gates action potential backpropagation in midbrain dopaminergic neurons. J Neurosci 27:1892-1901. CrossRef Medline

Gold C, Henze DA, Koch C, Buzsáki G (2006) On the origin of the extracellular action potential waveform: a modeling study. J Neurophysiol 95: 3113-3128. CrossRef Medline

González-Cabrera C, Meza R, Ulloa L, Merino-Sepúlveda P, Luco V, Sanhueza A, Oñate-Ponce A, Bolam JP, Henny P (2017) Characterization of the axon initial segment of mice substantia nigra dopaminergic neurons. J Comp Neurol 525:3529-3542. CrossRef Medline

Grace AA (1990) Evidence for the functional compartmentalization of spike generating regions of rat midbrain dopamine neurons recorded in vitro. Brain Res 524:31-41. CrossRef Medline

Grace AA, Bunney BS (1983a) Intracellular and extracellular electrophysiology of nigral dopaminergic neurons: 2. Action potential generating mechanisms and morphological correlates. Neuroscience 10:317-331. CrossRef Medline

Grace AA, Bunney BS (1983b) Intracellular and extracellular electrophysiology of nigral dopaminergic neurons: 1 . Identification and characterization. Neuroscience 10:301-315. CrossRef Medline

Grace AA, Bunney BS (1984) The control of firing pattern in nigral dopamine neurons: single spike firing. J Neurosci 4:2866-2876. CrossRef Medline

Harnett MT, Bernier BE, Ahn KC, Morikawa H (2009) Burst-timingdependent plasticity of NMDA receptor-mediated transmission in midbrain dopamine neurons. Neuron 62:826-838. CrossRef Medline

Häusser M, Stuart G, Racca C, Sakmann B (1995) Axonal initiation and active dendritic propagation of action potentials in substantia nigra neurons. Neuron 15:637-647. CrossRef Medline

Henny P, Brown MT, Northrop A, Faunes M, Ungless MA, Magill PJ, Bolam JP (2012) Structural correlates of heterogeneous in vivo activity of midbrain dopaminergic neurons. Nat Neurosci 15:613-619. CrossRef Medline

Henny P, Brown MT, Micklem BR, Magill PJ, Bolam JP (2014) Stereological and ultrastructural quantification of the afferent synaptome of individual neurons. Brain Struct Funct 219:631-640. CrossRef Medline

Henze DA, Borhegyi Z, Csicsvari J, Mamiya A, Harris KD, Buzsáki G (2000) Intracellular features predicted by extracellular recordings in the hippocampus in vivo. J Neurophysiol 84:390-400. CrossRef Medline

Hines ML, Carnevale NT (1997) The NEURON simulation environment. Neural Comput 9:1179-1209. CrossRef Medline

Hodgkin AL, Huxley AF (1952) A quantitative description of membrane current and its application to conduction and excitation in nerve. J Physiol 117:500-544. CrossRef Medline

Holt GR, Koch C (1999) Electrical interactions via the extracellular potential near cell bodies. J Comput Neurosci 6:169-184. CrossRef Medline

Hursh JB (1939) Conduction velocity and diameter of nerve fibers. Am J Physiol Leg Content 127:131-139. CrossRef

Jackson MB, Konnerth A, Augustine GJ (1991) Action potential broadening and frequency-dependent facilitation of calcium signals in pituitary nerve terminals. Proc Natl Acad Sci U S A 88:380-384. CrossRef Medline

Johnston DW, Wu S (1995) Foundations of cellular neurophysiology. Cambridge, MA: Massachusetts Institute of Technology.

Jun JJ, Steinmetz NA, Siegle JH, Denman DJ, Bauza M, Barbarits B, Lee AK, Anastassiou CA, Andrei A, Aydin C, Barbic M, Blanche TJ, Bonin V, Couto J, Dutta B, Gratiy SL, Gutnisky DA, Häusser M, Karsh B, Ledochowitsch P, et al. (2017) Fully integrated silicon probes for highdensity recording of neural activity. Nature 551:232-236. CrossRef Medline

Juraska JM, Wilson CJ, Groves PM (1977) The substantia nigra of the rat: a Golgi study. J Comp Neurol 172:585-600. CrossRef Medline

Kuhr WG, Wightman RM, Rebec GV (1987) Dopaminergic neurons: simultaneous measurements of dopamine release and single-unit activity 
during stimulation of the medial forebrain bundle. Brain Res 418:122128. CrossRef Medline

Kuznetsova AY, Huertas MA, Kuznetsov AS, Paladini CA, Canavier CC (2010) Regulation of firing frequency in a computational model of a midbrain dopaminergic neuron. J Comput Neurosci 28:389-403. CrossRef Medline

Lerner TN, Shilyansky C, Davidson TJ, Evans KE, Beier KT, Zalocusky KA, Crow AK, Malenka RC, Luo L, Tomer R, Deisseroth K (2015) Intactbrain analyses reveal distinct information carried by SNc dopamine subcircuits. Cell 162:635-647. CrossRef Medline

Lindén H, Hagen E, Leski S, Norheim ES, Pettersen KH, Einevoll GT (2014) LFPy: a tool for biophysical simulation of extracellular potentials generated by detailed model neurons. Front Neuroinform 7:41. CrossRef Medline

Liu PW, Blair NT, Bean BP (2017) Action potential broadening in capsaicin-sensitive DRG neurons from frequency-dependent reduction of Kv3 current. J Neurosci 37:9705-9714. CrossRef Medline

McCormick DA, Shu Y, Yu Y (2007) Neurophysiology: Hodgkin and Huxley model —still standing? Nature 445:E1-E2; discussion E2-E3. CrossRef Medline

Meza RC, López-Jury L, Canavier CC, Henny P (2018) Role of the axon initial segment in the control of spontaneous frequency of nigral dopaminergic neurons in vivo. J Neurosci 38:733-744. CrossRef Medline

Naundorf B, Wolf F, Volgushev M (2006) Unique features of action potential initiation in cortical neurons. Nature 440:1060-1063. CrossRef Medline

Ping HX, Shepard PD (1996) Apamin-sensitive $\mathrm{Ca}^{2+}$-activated $\mathrm{K}^{+}$channels regulate pacemaker activity in nigral dopamine neurons. Neuroreport 7:809-814. CrossRef Medline

Rall W, Shepherd GM (1968) Theoretical reconstruction of field potentials and dendrodendritic synaptic interactions in olfactory bulb. J Neurophysiol 31:884-915. CrossRef Medline

Rey HG, Pedreira C, Quian Quiroga R (2015) Past, present and future of spike sorting techniques. Brain Res Bull 119:106-117. CrossRef Medline

Safronov BV, Wolff M, Vogel W (2000) Excitability of the soma in central nervous system neurons. Biophys J 78:2998-3010. CrossRef Medline
Schweimer JV, Coullon GS, Betts JF, Burnet PW, Engle SJ, Brandon NJ, Harrison PJ, Sharp T (2014) Increased burst-firing of ventral tegmental area dopaminergic neurons in D-amino acid oxidase knockout mice in vivo. Eur J Neurosci 40:2999-3009. CrossRef Medline

Seutin V, Engel D (2010) Differences in $\mathrm{Na}^{+}$conductance density and $\mathrm{Na}^{+}$ channel functional properties between dopamine and GABA neurons of the rat substantia nigra. J Neurophysiol 103:3099-3114. CrossRef Medline

Silva NL, Pechura CM, Barker JL (1990) Postnatal rat nigrostriatal dopaminergic neurons exhibit five types of potassium conductances. J Neurophysiol 64:262-272. CrossRef Medline

Telenczuk B, Baker SN, Kempter R, Curio G (2015) Correlates of a single cortical action potential in the epidural EEG. Neuroimage 109:357-367. CrossRef Medline

Telenczuk M, Brette R, Destexhe A, Telenczuk B (2018) Contribution of the axon initial segment to action potentials recorded extracellularly. eNeuro 5:ENEURO.0068-18.2018. CrossRef Medline

Tepper JM, Martin LP, Anderson DR (1995) GABAA receptor-mediated inhibition of rat substantia nigra dopaminergic neurons by pars reticulata projection neurons. J Neurosci 15:3092-3103. CrossRef Medline

Terzuolo CA, Araki T (1961) An analysis of intra- versus extracellular potential changes associated with activity of single spinal motoneurons. Ann N Y Acad Sci 94:547-558. Medline

Tucker KR, Huertas MA, Horn JP, Canavier CC, Levitan ES (2012) Pacemaker rate and depolarization block in nigral dopamine neurons: a somatic sodium channel balancing act. J Neurosci 32:14519-14531. CrossRef Medline

Ungless MA, Grace AA (2012) Are you or aren't you? Challenges associated with physiologically identifying dopamine neurons. Trends Neurosci 35: 422-430. CrossRef Medline

Yang Y, Ramamurthy B, Neef A, Xu-Friedman MA (2016) Low somatic sodium conductance enhances action potential precision in time-coding auditory neurons. J Neurosci 36:11999-12009. CrossRef Medline

Yu Y, Shu Y, McCormick DA (2008) Cortical action potential backpropagation explains spike threshold variability and rapid-onset kinetics. J Neurosci 28:7260-7272. CrossRef Medline 